\title{
Josephson's junction, annulus maps, Birkhoff attractors, horseshoes and rotation sets
}

\author{
KEVIN HOCKETT AND PHIL I P HOL MES \\ Center for Applied Mathematics and Departments of Theoretical and \\ Applied Mechanics and Mathematics, Cornell University, Ithaca NY 18453, USA
}

(Received 7 March 1985 and revised 12 September 1985)

\begin{abstract}
We investigate the implications of transverse homoclinic orbits to fixed points in dissipative diffeomorphisms of the annulus. We first recover a result due to Aronson et al. [3]: that certain such 'rotary' orbits imply the existence of an interval of rotation numbers in the rotation set of the diffeomorphism. Our proof differs from theirs in that we use embeddings of the Smale [61] horseshoe construction, rather than shadowing and pseudo orbits. The symbolic dynamics associated with the non-wandering Cantor set of the horseshoe is then used to prove the existence of uncountably many invariant Cantor sets (Cantori) of each irrational rotation number in the interval, some of which are shown to be 'dissipative' analogues of the order preserving Aubry-Mather Cantor sets found by variational methods in area preserving twist maps. We then apply our results to the Josephson junction equation, checking the necessary hypotheses via Melnikov's method, and give a partial characterization of the attracting set of the Poincare map for this equation. This provides a concrete example of a 'Birkhoff attractor' [10].
\end{abstract}

\section{Introduction: forced oscillations, Poincaré maps and Birkhoff attractors}

The study of iterated mappings of the annulus or cylinder as dynamical systems began with Poincaré [56], [57], [58] and Birkhoff [7], [8], [9], who were interested in the three body problem of celestial mechanics and in the motion of a billiard ball in a simply connected domain $D \subset \mathbb{R}^{2}$ with convex boundary $\partial D$. Since the idealised billiard ball moves uniformly between impacts with $\partial D$, its state can be characterized by a succession of impact positions on $\partial D \cong S^{1}$ and reflection angles $\theta \in[0, \pi]=I$. Thus the motion may be described by a mapping $T: S^{1} \times I \rightarrow S^{1} \times I$. Here $A=S^{1} \times I$ is the annulus. In the case of perfectly elastic impacts, the system is conservative and the resulting mapping preserves area: $\operatorname{det}(D T)=1$. Katok [39] outlines a number of other examples which lead to area preserving mappings on annuli.

Birkhoff [10] subsequently generalized the problem somewhat to include dissipation and considered the case in which the map $T$ takes the annulus into its interior. Here det $(D T) \leq c<1$ and the resulting uniformly bounded contraction, coupled 
with the fact that $\boldsymbol{A}$ is a trapping region, allows one to define the attracting set

$$
\mathscr{A}=\mathrm{Cl}\left(\bigcap_{n \geq 0} T^{n}(A)\right),
$$

where $\mathrm{Cl}$ denotes closure. It is with the structure of such sets, loosely defined as Birkhoff attractors, that we shall be concerned with in this paper. Precise definitions will follow in $\$ 3$. See [30] for a modern treatment of some of Birkhoff's results.

Dissipative mappings on annuli arise in a number of other contexts. The main motivating example for the present work is the single point Josephson junction equation: a periodically forced second order differential equation which models certain aspects of a superconducting junction ([48], [43]). Suitably non-dimensionalized, the equation may be written as

or

$$
\ddot{x}+\delta \dot{x}+\sin x=\nu+\beta \cos \omega t,
$$

$$
\left.\begin{array}{rl}
\dot{\phi} & =r, \\
\dot{r} & =-\sin \phi+\nu-\delta r+\beta \cos \theta, \\
\dot{\theta} & =\omega .
\end{array}\right\} \quad \begin{aligned}
(\phi, r, \theta) & \in S^{1} \times \mathbb{R} \times S^{1} \\
& =A \times S^{1} .
\end{aligned}
$$

This equation has a simple mechanical analogue: the simple pendulum subject to linear damping $(\delta)$, constant torque $(\nu)$ and periodically varying torque $(\beta \cos \omega t)$. Levi et al. [43] present a complete analysis of the unforced case $(\beta=0)$ and a number of more recent studies have addressed aspects of the forced problem; see [59] and the references therein. Levi [42] has also recently studied the behaviour of a pair of coupled junctions subject to constant (non-periodic) forcing. A partial differential equation for the problem also exists: see [31] for studies of perturbations of this integrable infinite dimensional dynamical system.

For a periodically forced oscillator equation such as (1.3) we define a global cross section:

$$
\Sigma_{\theta_{0}}=\left\{(\phi, r, \theta) \in A \times S^{1} \mid \theta=\theta_{0} \in[0,2 \pi / \omega)\right\},
$$

and the associated time $-2 \pi / \omega$ or Poincaré return map:

$$
P_{\varepsilon}: \Sigma_{\theta_{0}} \rightarrow \Sigma_{\theta_{0}} \text {. }
$$

$P_{\varepsilon}=P_{\nu, \delta, \beta, \omega}$ is obtained by letting solutions of (1.3) based on $\boldsymbol{\Sigma}_{\theta_{0}}$ evolve for one period $2 \pi / \omega$ of the forcing function. Thus

$$
P(\phi, r)=\Pi \circ \Phi_{2 \pi / \omega}\left(\phi, r, \theta_{0}\right),
$$

where $\Phi_{t}:\left(\phi, r, \theta_{0}\right) \rightarrow(\phi(t), r(t), \theta(t))$ is the flow of (1.3) and $\Pi$ denotes projection onto the first factors $(\phi, r)$. We shall frequently drop the explicit parameter dependence in referring to maps such as $P$.

Another important motivating example is provided by a modification of the 'standard map' of Chirikov [21]:

$$
F_{\varepsilon, \gamma, \mu}:\left\{\begin{array}{l}
\phi \rightarrow \phi+r \\
r \rightarrow \varepsilon r+(1-\varepsilon) \mu-\gamma \cos (\phi+r)
\end{array} \quad(\phi, r) \in S^{1} \times \mathbb{R} .\right.
$$

Here $\varepsilon \in(0,1]$ is the dissipation parameter, $\gamma$ controls the strength of the nonlinearity and $\mu$ the position and frequency of the (smooth) invariant circle $r=\mu$ 
which exists for $\gamma=0$. The parameters $\varepsilon, \gamma$ and $\mu$ play roughly the same roles in (1.7) as do $\delta, \beta$ and $\nu$ in the Josephson equation. A map similar to (1.7) describes the dynamics of a ball bouncing under the influence of gravity on a vertically vibrating table (cf. [32]). We will return to the Josephson junction equation later in the paper.

The main purpose of the present work is to study the way in which Smale horseshoes ([61], [62]) are embedded in the non-wandering and attracting sets of maps like $P$ and $F$.

The presence of horseshoes, while not attractors themselves, is known to influence profoundly the structure of many candidates for strange attractors (cf. [26], [41], [64], [23], [25]). In the present case, we will show how the invariant Cantor sets (subshifts of finite type) associated with certain horseshoes, while only being part of the full attracting set, typically contain uncountably many invariant sets with specified irrational rotation numbers, along with periodic orbits, homoclinic and heteroclinic orbits and orbits for which rotation numbers do not exist. Thus we are able to provide a step towards understanding the global structure of Birkhoff attractors. Our work is closely related to that of Aubry [4], Aubry and LeDaeron [5], Mather [45], [46], [47], Katok [38], [39] and Boyland and Hall [13], who have worked extensively on order preserving orbits in area preserving, monotone twist maps of annuli. Very recently, LeCalvez [40] and Casdagli [16] have obtained results on dissipative monotone twist maps to which we refer in $\S 5$.

These authors use a Lagrangian variational formulation of the problem and concentrate on 'minimum energy' orbits. The Lagrangian ideas were originally suggested in this context by Percival [55]. Aubry et al. prove that the minimizing orbits and related minimax orbits are well ordered and that their closures are Cantor sets. Thus, when projected onto the phase variable ( $\phi$ in equation (1.7), for example) the orbits behave like those of a rigid rotation $\theta \rightarrow \theta+\alpha$, where $\alpha \in \mathbb{R} \backslash \mathbb{Q}$ is the rotation number of the Cantor set in question.

Our methods are rather different in spirit, and although we do find sets of order-preserving orbits whose closures are Cantor sets, we also show that our embedding of the horseshoe contains many badly ordered orbits.

Levi [41] and Aronson et al. [3] were among the first to bring recent developments in dynamical systems theory to bear on maps of the annulus, although it was the earlier work of Cartwright and Littlewood [15] and Levinson [44] on the van der Pol equation which originally inspired Smale's [61] construction of the horseshoe (cf. [62], [63]). Zehnder [67] considered the structure of area preserving maps near an elliptic fixed point in the generic case. Our first results on intervals of rotation numbers in $\$ 2$ are essentially the same as those of Levi and Aronson et al., although our proofs are different. In $\$ \S 3,4$ we ask more detailed questions on the structures of certain invariant (Cantor) subsets with specified rotation numbers suggested by the work of Aubry, Katok and Mather, although in our case we do not require the map to preserve area nor even that it be a monotone twist map. Our strategy in these three sections is to show carefully how the non-wandering set of the horseshoe, which results from the presence of transverse homoclinic orbits to 
a hyperbolic saddle point, is embedded in the annulus and then to work with the symbol sequences corresponding to certain orbits and collections of orbits. This bypasses a lot of the delicate analysis which would otherwise be necessary. Finally, we translate results on symbol sequences back into the corresponding results in the annular phase space. Although our conclusions are similar to those of Aubry, Katok and Mather (especially Mather [47]), we have not attempted to prove that our Cantor sets are the same as theirs in the case where both hypotheses are satisfied.

In $\S 5$ we apply our result to the first example introduced above. Using Melnikov's [49] method (cf. [23], [25]) we are able to prove that, for certain parameter ranges, the Poincare map of the Josephson equation (1.2)-(1.3) satisfies the hypotheses used in $\S \S 2,3,4$ and hence we can conclude the existence of non-trivial dynamics in the Josephson attracting set. Similar results can be obtained for the standard map (1.7) although we do not include them here. We illustrate our theorems with some numerical computations. Concluding remarks are given in $\S 6$.

We do not address here the important question of bifurcations: a description of how various components of the horseshoes (components with specified rotation numbers, say) appear and disappear as parameters are varied. While there is a reasonably good theory for maps on the circle (cf. [12], [52], [19], [20]), the case of annulus maps is still largely open, apart from the recent work of Chenciner [17], [18] on generalized Hopf bifurcation for maps. We are currently studying this question.

For a general introduction to the methods of dynamical systems and their application to non-linear oscillators and mappings such as (1.2)-(1.3) and (1.7), see [25] or [2].

\section{Homoclinic orbits, horseshoes and rotation numbers}

In this section we show how the presence of transverse intersections of stable and unstable manifolds implies the existence of invariant Cantor sets in which the dynamics are complicated (Smale horseshoes). Moreover, if the intersecting manifolds 'encircle' the annulus in a suitable manner, then the embedded horseshoe and its symbolic dynamical description can be chosen so as to reflect the rotation behaviour of orbits. The main result of this section, proposition 2.7 , is then used to prove a theorem due to Aronson et al. [3] on the existence of an interval of rotation numbers. The embedding of the horseshoe is 'natural' in that some iterate $f^{N}$ of $f$, restricted to the Cantor set, is conjugate to a full shift on two symbols. In subsequent sections we shall see that this does not generally yield order preserving (Birkhoff) orbits and that less usual embeddings will be required.

We start with some definitions and preliminaries:

2.1. Definitions. Let $f: A \rightarrow A$ be an orientation preserving $\left(C^{2}-\right)$ diffeomorphism of the annulus $A=S^{1} \times[a, b]$, where $S^{1}$ is the circle of length $2 \pi$ and $0 \leq a<b \leq \infty$. Let $\mathbf{x}=(\phi, r)$ denote a point in $A$ or in $\bar{A}=\mathbb{R} \times[a, b]$, its universal cover. Let $\bar{f}$ be a lift of $f$ to $\bar{A}$. Such lifts are unique up to $2 \pi$-translations $(\phi, r) \rightarrow(\phi+2 \pi, r)$. Let $\Pi_{1}: A \rightarrow S_{1}$ or $\Pi_{1}: \bar{A} \rightarrow \mathbb{R}$ denote projection onto the first factor: $\Pi_{1}(\phi, r)=\phi$. We denote a fixed (resp. $q$-periodic) point by $\mathbf{p}$ (resp. $\left.\mathbf{p}_{0}, \ldots, \mathbf{p}_{q-1}\right)$ and its stable and 
unstable manifolds by $W^{s}(\mathbf{p}), W^{u}(\mathbf{p})\left(\operatorname{resp} .\left\{W^{s}\left(\mathbf{p}_{j}\right)\right\}_{j=0}^{q-1},\left\{W^{u}\left(\mathbf{p}_{j}\right)\right\}_{j=0}^{q-1}\right)$. The subscript 'loc' denotes local manifolds: $W_{\text {loc }}^{u}(\mathbf{p})$, etc.

2.2. Rotation numbers. We define the (forward) rotation number of a point $\mathbf{x} \in A$ under $f$ as

$$
\rho(\mathbf{x})=\lim _{n \rightarrow \infty} \frac{1}{2 \pi n}\left[\Pi_{1} \circ \vec{f}^{n}(\mathbf{x})-\Pi_{1}(\mathbf{x})\right],
$$

where $\vec{f}$ is any lift of $f$, provided that the limit exists. $\rho(\mathbf{x})$ counts the average number of circuits of $\boldsymbol{A}$ made by the orbit $\left\{f^{n}(\mathbf{x})\right\}$ for each iteration of $f$. We also sometimes use the backward rotation number

$$
\rho^{-}(\mathbf{x})=\lim _{n \rightarrow \infty} \frac{1}{2 \pi n}\left[\Pi_{1} \circ \bar{f}^{-n}(\mathbf{x})-\Pi_{1}(\mathbf{x})\right] .
$$

2.3. Order preserving orbits. An orbit $\left\{f^{j}(\mathbf{x})\right\}_{j=-\infty}^{\infty}$ of $f$ is said to be order preserving if for any lift, $\bar{f}$ and any two points $\mathbf{y}, \mathbf{y}^{\prime} \in\left\{\bar{f}^{j}(\mathbf{x})\right\}$ we have

$$
\Pi_{1}(\mathbf{y})<\Pi_{1}\left(\mathbf{y}^{\prime}\right) \Rightarrow \Pi_{1}(\bar{f}(\mathbf{y}))<\Pi_{1}\left(\bar{f}\left(\mathbf{y}^{\prime}\right)\right) .
$$

The projection $\left\{\Pi_{1}\left(f^{j}(\mathbf{x})\right)\right\}$ of an order preserving orbit onto $S^{1}$ has the same order properties as the orbits of the rigid rotation $r_{\alpha}: S^{1} \rightarrow S^{1}$ defined by $\phi \rightarrow \phi+\alpha$. Thus order preserving orbits always possess rotation numbers. Order preserving periodic orbits are also sometimes called ( $p / q$-) Birkhoff orbits (cf. [38], [28]).

2.4. Symbolic dynamics. Let $\Sigma=\{0,1\}^{\mathbf{Z}}$ denote the space of biinfinite sequences of the two symbols 0,1 and $\Sigma^{+}=\{0,1\}^{\mathbf{Z}^{+}}$denote the space of semi-infinite sequences. Let $\sigma: \Sigma \rightarrow \Sigma$ denote the shift map, operating on a sequence $\mathbf{a}=\left\{a_{j}\right\}_{j=-\infty}^{\infty} \in \Sigma$ as follows:

$$
(\sigma(\mathbf{a}))_{j}=a_{j+1}
$$

We endow $\Sigma$ with the metric

$$
d(\mathbf{a}, \mathbf{b})=\sum_{j=-\infty}^{\infty} \frac{\left|a_{j}-b_{j}\right|}{2^{j j \mid}} .
$$

If the block $\left(a_{j}, \ldots, a_{j+N}\right)$ is repeated indefinitely in the past (resp. future) we denote it with a superscript - to the left (resp. + to the right). Thus $\cdots 000 \cdot 000 \cdots=$ ${ }^{-}(0)^{+}, \cdots 001 \cdot 011 \cdots={ }^{-}(0) 1 \cdot 0(1)^{+}$, etc. The (forward) rotation number of a symbol sequence $\mathrm{a} \in \mathrm{\Sigma}$ is defined as

$$
R(\mathbf{a})=\lim _{n \rightarrow \infty} \frac{1}{n} \sum_{j=0}^{n-1} a_{j}
$$

if the limit exists. Similarly,

$$
R^{-}(\mathbf{a})=\lim _{n \rightarrow \infty} \frac{1}{n} \sum_{j=0}^{n-1} a_{-j}
$$

is the backward rotation number. $\Sigma^{+}$is endowed with a metric like that of (2.3); we just replace the lower limit of summation by 0 , and $R(\mathbf{a})$ for $\mathbf{a} \in \Sigma^{+}$is also defined by (2.4a). Of course, $R^{-}$is not defined on $\Sigma^{+}$.

2.5. Attracting sets. Suppose that the annulus $A=S^{1} \times[a, b]$ contains an annular trapping region $B=S^{1} \times[c, d], a \leq c<d \leq b$. This means that $f(B) \subset$ int $(B)$ (int 
denotes interior). Thus the set

$$
\mathscr{A}=\bigcap_{n=0}^{\infty} f^{n}(B)
$$

is non-empty. We call $\mathscr{A}$ the attracting set for the trapping region $B$. If, as in our examples, $\operatorname{det}(D F) \leq c<1$, then $f^{n}$ decreases areas at least by $c^{n}$ and thus $\mathscr{A}$ has zero area. If $\mathscr{A}$ contains transverse homoclinic points, we call it a strange attracting set, and if it contains a dense orbit we call it a strange attractor. See [25, $\$ 5.4-5]$ for more details.

We shall also rather loosely refer to $\mathscr{A}$ as a Birkhoff attracting set or Birkhoff attractor (cf. [10]).

We recall that a set $S$ is minimal if it is invariant under $f$ and closed, and no proper subset of $S$ has the latter properties. Thus, while an attractor is minimal, a Birkhoff attracting set may not be.

2.6. Rotary homoclinic points. We suppose that $f$ has a hyperbolic fixed point $\mathbf{p}$ whose stable and unstable manifolds intersect transversely at some point $q \neq \mathbf{p}$. We will require the intersecting manifolds to encircle the annulus $A$. To define this notion, fix a neighbourhood $U$ of $p$ and the local stable and unstable manifolds $W_{\text {loc }}^{s}(\mathbf{p}), W_{\text {loc }}^{u}(\mathbf{p})$. Let $k, l \in \mathbb{Z}^{+}$be the smallest integers such that the set $W=$ $f^{\prime}\left(W_{\mathrm{loc}}^{u}(\mathbf{p})\right) \cap f^{-k}\left(W_{\mathrm{loc}}^{s}(\mathbf{p})\right)$ contains q. Then if $f^{l}\left(W_{\mathrm{loc}}^{u}(\mathbf{p})\right) \cup f^{-k}\left(W_{\mathrm{loc}}^{s}(\mathbf{p})\right) \cup\{\mathbf{p}\}$ contains a closed curve which encircles the annulus at least once we say that the orbit $\left\{f^{j}(q)\right\}_{j=-\infty}^{\infty}$ is a rotary (transverse) homoclinic orbit. We note that, for such an orbit,

$$
\sum_{j=-\infty}^{\infty} \Pi_{1} \circ \bar{f}^{j}(\mathbf{q})=2 \pi K \quad \text { for some } K \geq 1,
$$

i.e. rotary homoclinic orbits travel $K$ times around the annulus. If $K=1$ we call the orbit simple or 1-rotary, otherwise it is K-rotary; see figure 1. The term 'rotary' is borrowed from literature on the Josephson and pendulum equations.

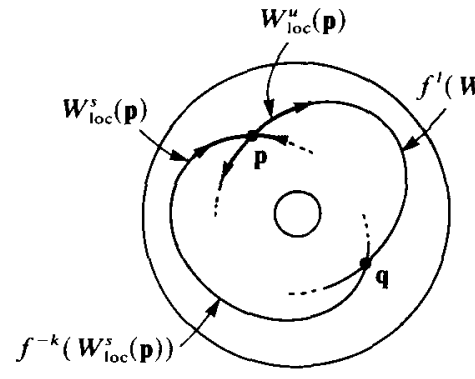

(a) a simple rotary homoclinic orbit

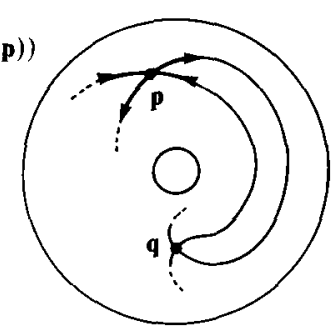

(b) a non-rotary homoclinic orbit

FIGURE 1. Rotary homoclinic orbits.

Analogous notions hold for homoclinic orbits to periodic points $\left(\mathbf{p}_{0}, \ldots, \mathbf{p}_{q-1}\right)$ having rotation number $p / q$. However, all we need assume to guarantee rotary homoclinic orbits in this case is that $W^{u}\left(\mathbf{p}_{0}\right)$ intersects $W^{s}\left(\mathbf{p}_{j}\right)$ transversely at $\mathbf{q} \neq \mathbf{p}_{k}$ 
for some $0<j \leq q-1$. This implies that $W^{u}\left(\mathbf{p}_{k}\right) \cap W^{s}\left(\mathbf{p}_{j+k}\right) \neq \varnothing$ for all $k$ and hence that a rotary homoclinic cycle can be constructed by piecing together $q$ heteroclinic orbits $\left\{f^{j q}(\mathbf{q})\right\}_{j=-\infty}^{\infty},\left\{f^{j(q+1)}(\mathbf{q})\right\}_{j=-\infty}^{\infty}$, etc. Such an orbit will be $K$-rotary for $f^{q}$.

It is well known that the presence of transverse homoclinic orbits implies complicated dynamics, positive topological entropy and, loosely speaking, chaotic dynamics. More precisely, the Smale-Birkhoff homoclinic theorem shows that a map $f$ with a transverse homoclinic point possesses a non-wandering Cantor set $\Lambda$ on which $f$ is conjugate to a subshift of finite type. Equivalently, there is some integer $N<\infty$ and a subset $\Lambda^{N} \subset \Lambda$ such that $\left.f^{N}\right|_{\Lambda^{N}}$ is conjugate to a full shift on two symbols (cf. [61], [50], [25]). In proving the following result we pay particular attention to the geometry of $\Lambda$ relative to $A$ and the symbolic dynamics of $f^{N}$ on $\Lambda^{N}$.

2.7. Proposition. Suppose that $f: A \rightarrow A$ has a hyperbolic fixed (or periodic) point $\mathbf{p}$ with a K-rotary transverse homoclinic orbit (or cycle). Then there exists a hyperbolic non-wandering Cantor set $\Lambda \subset A$, an integer $N<\infty$, a subset $\Lambda^{N} \subset \Lambda$ and a homeomorphism $h: \Lambda^{N} \rightarrow \Sigma$ such that $\left.f^{N}\right|_{\Lambda^{N}}=h^{-1} \circ \sigma \circ h$. Moreover, $\Lambda$ and $\Lambda^{N}$ can be chosen so that the symbol sequences $\mathbf{a}=h(\mathbf{x})$ of points $\mathbf{x} \in \Lambda^{N}$ reflect the rotation behaviour; in fact for $\mathbf{x} \in \Lambda^{N}$ we have $\rho(\mathbf{x})=(K / N) R(h(\mathbf{x}))$ if either limit exists and if $\rho(\mathbf{x})$ does not exist then nor does $R(h(\mathbf{x}))$.

Proof. We will only prove the theorem for the case of a fixed point. The construction for the periodic case is similar in spirit but more involved. We first construct a Markov partition of $N$ disjoint 'rectangles'; this construction is the one used in [25, theorem 5.3.5]. Let $U$ denote a neighbourhood of $\mathbf{p}$. Fix a point $\mathbf{q} \neq \mathbf{p}$ on the homoclinic orbit and pick the smallest integers $k$, $l$ for which $q \in V=f^{l}(U) \cap f^{-k}(U)$. By adjusting the size of $U$ if necessary, we can ensure that $V$ has only one component, that the images $f^{j}(V),-l<j<k$, are disjoint and that $f^{-l}(V)$ and $f^{k}(V)$ lie in $U$ and intersect in a single component. Moreover, since the orbit of $\mathbf{q}$ encircles $A$ precisely $K$ times, the images $f^{j}(V), 0 \leq j \leq N$ make $K$ full circuits of $A$ also.

Let $w^{u}$ and $w^{s}$ denote the pieces of $W^{u}(\mathbf{p}) \cap V, W^{s}(\mathbf{p}) \cap V$ containing $\mathbf{q}$. The lambda-lemma of Palis [54] (cf. [51]) shows that the iterates $f^{j}\left(w^{u}\right)$ approach $W_{\text {loc }}^{s}(\mathbf{p})$ and iterates $f^{-j}\left(w^{s}\right)$ approach $W_{\text {loc }}^{u}(\mathbf{p})$ as $j \rightarrow \infty$; moreover, the tangent spaces to those curves also approach one another. Thus, by taking $U$ smaller (and $k, l$ larger), we can ensure that the images $f^{k}(V)$ and $f^{-1}(V)$ are thin strips in $U$ almost parallel to $W_{\text {loc }}^{u}(\mathbf{p})$ and $W_{\mathrm{loc}}^{\text {s }}(\mathbf{p})$ respectively; see figure $2(\mathrm{a})$. We pick a further thin strip $V_{0} \subset U$ bounded on two sides by the local stable and unstable manifolds of $\mathbf{p}$ and thin enough in the $W^{u}(\mathbf{p})$ direction so that $f^{j}\left(V_{0}\right) \subset U$ for all $0 \leq j \leq k+l$ and $f^{k+l}\left(V_{0}\right)$ stretches across $U$ along $W_{\text {loc }}^{u}(\mathbf{p})$ so that it crosses $f^{-I}(V)$. Letting $N=k+l$ and setting $H_{0}=f^{N}\left(V_{0}\right)$ and picking $V_{1} \subset f^{-l}(V), H_{1} \subset f^{k}(V)$, we have two strips $V_{i}, i=0,1$, each of whose images $H_{i}$ under $f^{N}$ intersect both $V_{0}$ and $V_{1}$ in the manner necessary for the horseshoe construction. In particular, the estimates on $D f^{N}$ necessary for Moser's [50] proof of hyperbolicity will be satisfied if $N$ is large enough, since $D f^{N}$ is dominated by the linearization of $f$ near the hyperbolic fixed point $\mathbf{p}$; see figure 2(b). 


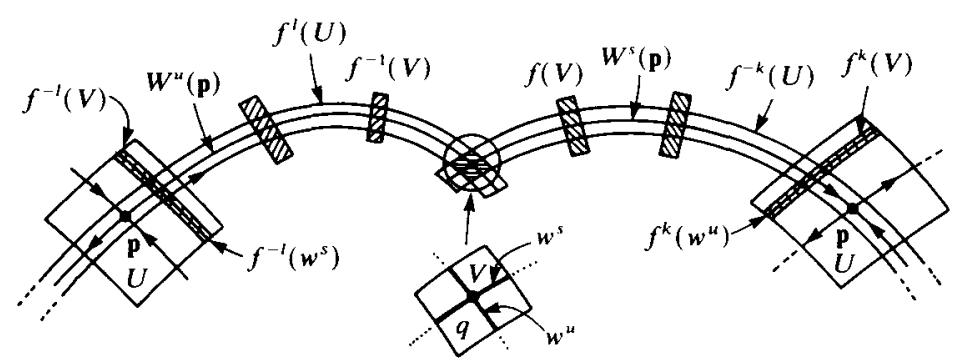

(a) the sets $U$ and $f^{j}(V),-l \leq j \leq k$

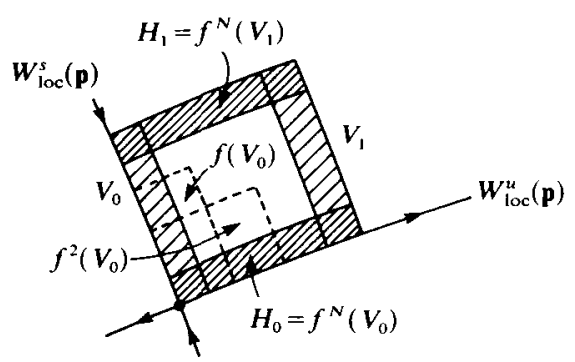

(b) the sets $V_{0}, V_{1}, H_{0}$ and $H_{1}$

FIGURE 2. Rectangles and horseshoes for $f: A \rightarrow A$, shown in covering space.

To conclude the proof of the first part of the theorem, we define the Cantor sets

$$
\begin{aligned}
\Lambda^{N} & =\bigcap_{j=-\infty}^{\infty} f^{j N}\left(V_{0} \cup V_{1}\right), \\
\Lambda & =\bigcup_{j=0}^{N-1} f^{j}\left(\Lambda^{N}\right) .
\end{aligned}
$$

That $\Lambda^{N}$ is a Cantor set follows from the nesting property of the images $f^{-n N}\left(V_{i}\right) \cap V_{j}$ and $f^{n N}\left(H_{i}\right) \cap H_{j}, n=1,2, \ldots$ (cf. [50, §3]). To each point $\mathrm{x} \in \Lambda^{N}$ we assign a bi-infinite symbol sequence $h(\mathbf{x})$ by the rule

$$
h_{j}(\mathbf{x})=\left\{\begin{array}{ll}
0 & \text { if } f^{j N}(\mathbf{x}) \in V_{0} \\
1 & \text { if } f^{j N}(\mathbf{x}) \in V_{1}
\end{array},\right.
$$

where $h_{j}$ denotes the $j$ th symbol of $h(\mathbf{x})$. Since the intersections $H_{i}=f^{N}\left(V_{i}\right) \cap V_{j}$ are all non-empty, every symbol sequence $a \in \Sigma$ is realized by a point $h^{-1}(\mathbf{a}) \in \Lambda^{N}$. In fact, using the metric (2.3) on $\Sigma$ and the natural Euclidean metric on $\Lambda^{N}$ inherited from its embedding in $A, h$ is easily seen to be a homeomorphism (see $[50, \S 3]$ or $[25, \S 5.1])$.

We now show that $h: \Lambda^{N} \rightarrow \Sigma$ preserves rotation information, thus completing the proof. First note that if $\mathbf{x}=\mathbf{p}$ then $f^{i}(\mathbf{x}) \in V_{0}$ for all $i$ and thus $h(\mathbf{x})=$ $\cdots 000 \cdot 000 \cdots={ }^{-}(0)^{+}$. Thus from equation (2.4) $R(\mathbf{x})=0$, and since $\mathbf{p}$ is fixed for $f$ the result holds trivially. On the other hand, if $h(\mathbf{x})=\cdots 111 \cdot 111 \cdots={ }^{-}(1)^{+}$ then $f^{i N}(\mathbf{x}) \in V_{1}$ for all $i$ and $f^{i N+j}(\mathbf{x}) \in f^{i}\left(V_{1}\right)=f^{j-l}(V)$ for $0<j<N$. Thus, after $N$ iterates $\mathrm{x}$ has travelled $K$ times around the annulus:

$$
\Pi_{1} \circ \bar{f}^{N}(\mathbf{x})-\Pi_{1}(\mathbf{x})=2 \pi K \text {. }
$$


This shows that the fixed point $\mathbf{r}=h^{-1}\left({ }^{-}(1)^{+}\right)$for $f^{N}$ is in fact an $N$-periodic point with rotation number $\rho(\mathbf{r})=K / N$ for $f$. Thus the result holds in this case also. Similarly any sequences $\mathbf{a}=a_{-j} \cdots a_{0} \cdots a_{k}(0)^{+}$or $\cdots a_{-j} \cdots a_{0} \cdots a_{k}(1)^{+} \cdots$ with common forward asymptotic behaviours correspond to points $h^{-1}(a)$ with $\rho(\mathbf{x})=0$ or $K / N$ respectively. Such points lie in $W^{s}(\mathbf{p})$ and $W^{s}(\mathbf{r})$ respectively. Henceforth we can assume that the right hand part of $h(\mathbf{x})$ contains only finite (though possibly arbitrarily long) blocks of consecutive 0's or 1's.

More generally, from figure 2 it is clear that each entry ' 1 ' in the sequence $h(\mathbf{x})$ corresponds to an image of $\mathrm{x}$ in $V_{1}$ which will travel $K$ times around $A$ in the next $N$ iterates of $f$, while each entry ' 0 ' corresponds to a point in $V_{0}$ which will mark time in $U$ for the next $N$ iterates.

Now assume that $\rho(\mathbf{x})$ exists, so that given $\varepsilon>0$ there exists $M$ such that $k \geq M$ implies that

$$
\left.\mid \frac{1}{2 \pi N k}\left[\Pi_{1} \circ \bar{f}^{N k}(\mathbf{x})-\Pi_{1}(\mathbf{x})\right]-\rho(\mathbf{x})\right] \mid<\varepsilon / 2 .
$$

Now $\Pi_{1} \circ \bar{f}^{N k}(\mathbf{x})=\Pi_{1}(\mathbf{x})+2 \pi m_{k}+\alpha_{k}$ for some $m_{k} \in \mathbb{Z}^{+}$and $\alpha_{k} \in[0,2 \pi)$. Thus $k \geq M$ implies that

$$
\begin{gathered}
\left|\frac{2 \pi m_{k}+\alpha_{k}}{2 \pi N k}-\rho(\mathbf{x})\right|<\frac{\varepsilon}{2}, \\
\left|\rho(\mathbf{x})-\frac{m_{k}}{N k}\right|-\frac{\alpha_{k}}{2 \pi N k} \leq\left|\rho(\mathbf{x})-\left(\frac{m_{k}}{N k}+\frac{\alpha_{k}}{2 \pi N k}\right)\right|<\frac{\varepsilon}{2},
\end{gathered}
$$

or

$$
\left|\rho(\mathbf{x})-\frac{m_{k}}{N k}\right|<\frac{\varepsilon}{2}+\frac{\alpha_{k}}{2 \pi N k} .
$$

Since $0 \leq \alpha_{k}<2 \pi$ we can pick $M^{\prime} \geq M$ sufficiently large such that, for $k \geq M^{\prime}$ :

$$
\left|\rho(\mathbf{x})-\frac{m_{k}}{N k}\right|<\varepsilon .
$$

Now $m_{k}$ is just the number of full turns of $A$ executed by $\mathbf{x}$ in $N k$ iterates. Thus, from the remarks above,

$$
m_{k}=K \sum_{j=0}^{k-1} a_{j}
$$

and so from $(2.12)$

$$
\left|\rho(\mathbf{x})-\frac{K}{N k} \sum_{j=0}^{k} a_{j}\right|<\varepsilon
$$

Thus we obtain

$$
\rho(\mathbf{x})=\frac{K}{N} R(h(\mathbf{x}))
$$

as required. 
Conversely, suppose $\gamma=(K / N) \lim _{k \rightarrow \infty} 1 / k \sum_{j=0}^{k-1} a_{j}$ exists. Then, reversing the steps above, we see that $\gamma$ satisfies

$$
\lim _{k \rightarrow \infty}\left|\gamma-\frac{1}{2 \pi N k}\left[\Pi_{1} \circ f^{N k}(\mathbf{x})-\Pi_{1}(\mathbf{x})\right]\right|=0
$$

i.e. $\rho(x)=\gamma$ exists.

This argument shows that either both limits exist and are equal or neither limit exists.

2.8 Theorem. (Aronson et al. [3]) Suppose that $f: A \rightarrow A$ has a hyperbolic fixed point with a K-rotary transverse homoclinic orbit. Then there exists a non-trivial closed interval $I \subset \mathbb{R}$ containing 0 such that for every $\alpha^{+}, \alpha^{-} \in I$ there are uncountably many points $\mathbf{x} \in A$ with forward rotation number $\rho(x)=\alpha^{+}$and uncountably many points with backward rotation number $\alpha^{-}$. Moreover there are uncountably many points $\mathbf{x} \in A$ for which neither forward nor backward rotation numbers exist, and uncountably many points $\mathbf{x} \in A$ whose backward and forward rotation numbers $\rho^{-}(\mathbf{x})=\alpha^{-}, \rho^{+}(\mathbf{x})=\alpha^{+}$ both exist (and $\alpha^{-}, \alpha^{+} \in I$ can be chosen arbitrarily).

Proof. In view of proposition 2.7 we need only exhibit symbol sequences with the correct asymptotic behaviours. We deal with periodic points first. We have already constructed sequences $\cdots(0)^{+}$and $\cdots(1)^{+}$with $R(a)=0$ and 1 , corresponding to points with $\rho(\mathbf{x})=0$ and $K / N$ respectively.

The interval $I$ of the theorem will be $[0, K / N]$. Pick $\alpha^{+}=p / q \in(0, K / N)$ where $p, q \in \mathbb{Z}^{+}$and $(p, q)=1$. Let $a, b$ be non-negative integers such that

$$
\frac{a \cdot 0+b \cdot(K / N)}{a+b}=p / q \text { or } \frac{a}{b}=\frac{K q}{N p}-1
$$

There are countably many solutions to this equation, for pick any $b \in \mathbb{Z}^{+}$such that $((K q / N p)-1) b \in \mathbb{Z}^{+}$and let $a=((K q / N p)-1) b$ (recall that $p / q<K / N$, so $(K q / N p)-1>0)$. Form a sequence $c$ by repeating blocks of length $a+b$ each containing $a$ 's and $b 1$ 's. It is clear that $R(\mathrm{c})=N p / K q$ and $\rho\left(h^{-1}(\mathrm{c})\right)=p / q$. (Note that the resulting orbits $\left\{f^{j}\left(h^{-1}(\mathrm{c})\right)\right\}$ generally have periods which are multiples of $q$, e.g. $h^{-1}\left({ }^{-}(01)^{+}\right)$has $\rho=K / 2 N$ and is $2 N$ periodic while $h^{-1}\left(-(0011)^{+}\right)$and $h^{-1}\left({ }^{-}(010110)^{+}\right)$also have $\rho=K / 2 N$ but are $4 N$ and $6 N$ periodic respectively). Such orbits have the same forward and backward rotation numbers.

To construct uncountably many orbits with specified forward or backward rotation number $\rho(x)=p / q$ we choose a sequence $c_{0} \cdots c_{k}$ of length $(a+b)$ as above and form sequences $\cdots\left(c_{0} \cdots c_{k}\right)^{+}$and ${ }^{-}\left(c_{0} \cdots c_{k}\right) \cdots$ with arbitrary tails to the left and right respectively. To construct orbits with different rotation behaviours in the past and future pick integers $p / q, p^{\prime} / q^{\prime} \in[0, K / N]$ and form two distinct sequences of length $a+b$ and two of length $a^{\prime}+b^{\prime}\left(a^{\prime}=\left(\left(K q^{\prime} / N p^{\prime}\right)-1\right) b^{\prime}\right)$ as above, denoting these sequences by $\mathbf{c}_{0}, \mathbf{c}_{1} ; \mathbf{c}_{0}^{\prime}, \mathbf{c}_{1}^{\prime}$. Then the set of orbits of the form $h^{-1}\left(\cdots \mathbf{c}_{i_{-2}} \mathbf{c}_{i_{-1}} \cdot \mathbf{c}_{i_{0}}^{\prime} \mathbf{c}_{i_{1}}^{\prime} \cdots\right)$, where $i_{j}=0$ or 1 , will have the desired behaviour. In particular, there are uncountably many such orbits because the $c_{i_{j}}$ 's and $\mathbf{c}_{i_{j}}^{\prime}$ 's can be chosen arbitrarily from the sets $\left\{\mathbf{c}_{0}, \mathbf{c}_{1}\right\}^{\mathbf{Z}^{+}}$and $\left\{\mathbf{c}_{0}^{\prime}, \mathbf{c}_{1}^{\prime}\right\}^{\mathbf{Z}^{+}}$respectively. An arbitrary finite central block of 0 's and 1 's can also be added. 
To construct an orbit whose rotation number does not exist, we choose a sequence a which is dense in $\Sigma$. For example, join end to end all possible finite blocks of 0 's and 1's of length $1,2, \ldots$. There are clearly uncountably many such sequences.

Finally we construct orbits with irrational rotation numbers. Intuitively one just requires that the 'average' proportion of 1's is irrational (cf. [41, pp. 70-72]), but we must ensure that the limit $R(a)$ of equation (2.4) exists. To construct sequences a with specified $R(\mathbf{a})=\alpha \in[0,1] \backslash \mathbb{Q}$ we consider the rigid rotation $\theta \mapsto r_{\alpha}(\theta)=\theta+\alpha$ of the circle $S^{1}$ of length 1. Fix the origin $0 \in S^{1}$ and the point $\alpha \in S^{1}$ and define the arcs $A_{1}=[0, \alpha)$ and $A_{0}=[\alpha, 0)$ of lengths $\alpha$ and $1-\alpha$ respectively. Choose any point $\theta_{0} \in S^{1}$ and consider its orbit $\left\{r_{\alpha}^{j}\left(\theta_{0}\right)\right\}_{j=0}^{\infty}$. Define $a\left(\theta_{0}\right)$ by

$$
a_{j}\left(\theta_{0}\right)= \begin{cases}0 & \text { if } r_{\alpha}^{j}\left(\theta_{0}\right) \in A_{0}, \\ 1 & \text { if } r_{\alpha}^{j}\left(\theta_{0}\right) \in A_{1} .\end{cases}
$$

Since the orbit of $\theta_{0}$ is countably dense in $S^{1}$, as $\theta_{0}$ ranges over $S^{1}$ uncountably many distinct sequences are created (in fact there is a $1: 1$ correspondence between points $\theta_{0} \in S^{1}$ and sequences $\left.\mathbf{a}\left(\theta_{0}\right)\right)$. Now the 'conventional' rotation number of any point $\theta_{0}$ under $r_{\alpha}$ is $\alpha$ and so all we need do is show that $R\left(a\left(\theta_{0}\right)\right)=$ $\lim _{n \rightarrow \infty} 1 / n \sum_{j=0}^{n} a_{j}=\alpha$, i.e. $R$ is a correct measure of the circular rotation number. But this is precisely the content of proposition 6.2.1 of [25], where the rotation number for homeomorphisms of the circle is defined intrinsically rather than using lifts (also see lemma E, $\S 3.3$, below).

Given such a sequence $\mathrm{a}=a_{0} a_{1} \cdots$ we let $a_{-j}=a_{j}$ and can thus construct uncountably many sequences with forward and backward rotation numbers $\alpha$, and hence uncountably many points $x$ with $\rho(x)=\rho^{-}(\mathbf{x})=K \alpha / N \in[0, K / N] \backslash Q$. To construct orbits with differing forward and backward rotation numbers we simply piece together left-going and right-going sequences constructed using rigid rotations $r_{\alpha^{\prime}}$ and $r_{\alpha}$. The central (finite) connecting portion is arbitrary, as in the case of periodic orbits.

Remarks. Although here we have only given the theorem for the case of a fixed point with a homoclinic orbit, an analogue for a $q$-periodic point of rotation number $p / q$ exists. In this case the interval $I=\left[p / q, p^{\prime} / q^{\prime}\right]$ where $\left|p / q-p^{\prime} / q^{\prime}\right|=\mathcal{O}(1 / N)$ is determined by the number of iterates necessary to ensure hyperbolicity for $D f^{N}$. In specific cases $N$ can be estimated, see $\S 5$.

In the next section we will develop the irrational rotation idea used above to construct orbits with irrational rotation numbers whose closures are Cantor sets. Some of these correspond to the order preserving (Aubry-Mather) Cantor sets referred to in $\S 1$. However, this requires a more careful study of the global structure of the embedded manifolds $W^{u}(\mathbf{p}), W^{s}(\mathbf{p}) \subset A$, which we carry out in $\S 4$.

\section{Irrational Cantor sets in the horseshoe}

We now use the symbolic description of orbits developed in proposition 2.7 to characterize certain classes of orbits in the non-wandering sets $\Lambda^{N}$ and $\Lambda$ defined in (2.7). This section is devoted to the proof of: 
3.1. THEOREM. Suppose that $f: A \rightarrow A$ has a K-rotary transverse homoclinic point $\mathbf{q} \in W^{u}(\mathbf{p}) \cap W^{s}(\mathbf{p})$ to a hyperbolic fixed point $\mathbf{p}$. Then there exists an integer $N<\infty$ such that for any $\gamma \in[0, K / N]-\mathbb{Q}$ there exist uncountably many disjoint invariant Cantor sets $\Lambda_{\gamma}$ such that $\rho(\mathbf{x})=\rho^{-}(\mathbf{x})=\gamma$ for all $\mathbf{x} \in \Lambda_{\gamma} \Lambda_{\gamma}=\Lambda_{\gamma}(\mu)$ can be parameterized (with some redundancy) by $\mu \in(0,1]$.

3.2. Remarks. The sets $\Lambda_{\gamma}(\mu)$ are all contained in the Cantor set $\Lambda$ of proposition 2.7. In fact each $\Lambda_{\gamma}(\mu)=\bigcup_{j=0}^{N-1} f^{j}\left(\Lambda_{\gamma}^{N}(\mu)\right)$, for a set $\Lambda_{\gamma}^{N}(\mu) \subset \Lambda^{N}$ which we will construct below. The majority of these Cantor sets are not order preserving, see $\S 4$. They appear to be analogues of some of the sets for area preserving maps recently constructed by Mather [47].

To prove the theorem we will further develop Hedlund's [29] construction of invariant Cantor sets, already alluded to in the proof of theorem 2.8, above. Although the basic idea is Hedlund's, we divide the circle into four arcs rather than two and are thus using a construction similar to the double cover suggested by Boyland [12].

3.3. Irrational Cantor sets. We first construct symbol sequences of rotation number $\alpha=\gamma N / K \in(0,1)$ for specified $\gamma \in[0, K / N] \backslash \mathbb{Q}$. Let $r_{\alpha}$ denote the rigid rotation $\theta \mapsto \theta+\alpha$ defined on the circle $S^{1}$ of circumference 1. Fix $c \in(0,1-\alpha]$ and divide the circle into four arcs:

$$
A_{0}(c)=[0, c) ; \quad B_{0}(c)=\left[c, c+\frac{\alpha}{2}\right) ; \quad A_{1}(c)=\left[c+\frac{\alpha}{2}, 1-\frac{\alpha}{2}\right) ; \quad B_{1}(c)=\left[1-\frac{\alpha}{2}, 1\right)
$$

To each irrational $\alpha \in(0,1)$, each point $x \in S^{1}$ and real number $c \in[0,1-\alpha)$, we assign a bi-infinite symbol sequence $\mathbf{a}_{\alpha}(x, c) \in \Sigma$ with entries $a_{\alpha}^{i}(x, c)$ determined by

$$
a_{\alpha}^{i}(x, c)= \begin{cases}0 & \text { if } r_{\alpha}^{i}(x) \in A_{0}(c) \cup A_{1}(c), \\ 1 & \text { if } r_{\alpha}^{i}(x) \in B_{0}(c) \cup B_{1}(c) .\end{cases}
$$

In an analogous fashion we define sequences $\mathbf{a}_{\alpha}^{\prime}(x, c)$ using the partition $A_{0}^{\prime}(c)=$ $(0, c], \ldots, B_{1}^{\prime}(c)=(1-(\alpha / 2), 1]$.

We now state and prove several lemmas, which will together imply theorem 3.1.

LemMA A. $x=y \Leftrightarrow \mathbf{a}_{\alpha}(x, c)=\mathbf{a}_{\alpha}(y, c)\left(\right.$ resp. $\left.\mathbf{a}_{\alpha}^{\prime}(x, c)=\mathbf{a}_{\alpha}^{\prime}(y, c)\right)$.

Proof. $(\Rightarrow)$ immediate, from the definitions.

$(\Leftarrow)$ Suppose that $\mathbf{a}_{\alpha}(x, c)=\mathbf{a}_{\alpha}(y, c)$ but $x \neq y$, so that $|x-y|=\varepsilon$ for some $\varepsilon>0$. Pick $x^{\prime} \in A_{0}(c) \cup A_{1}(c)$ and $y^{\prime}=x^{\prime}+|x-y| \in B_{0}(c) \cup B_{1}(c)$ such that $x^{\prime}$ and $y^{\prime}$ each lie $\varepsilon / 2$ from the nearest boundary of $A_{i}$ or $B_{i}$. Since the irrational $r_{\alpha}$-orbit $\left\{r_{\alpha}^{n}(x)\right\}_{n=-\infty}^{\infty}$ is dense in $S^{1}$ for any $x \in S^{1}$, there exists $N<\infty$ such that $\left|r^{N}(x)-x^{\prime}\right|<\varepsilon / 4$, and, since the distance $\left|r_{\alpha}^{k}(x)-r_{\alpha}^{k}(y)\right|$ between images of $x$ and $y$ is preserved under rigid rotation, we also have $\left|r_{\alpha}^{N}(y)-y^{\prime}\right|<\varepsilon / 4$. Thus $a_{\alpha}^{N}(x, c)=0$ while $a_{\alpha}^{N}(y, c)=1$, so that $\mathbf{a}_{\alpha}(x, c) \neq \mathbf{a}_{\alpha}(y, c)$, providing a contradiction. The proof for $\mathbf{a}^{\prime}$ is the same.

LeMMA B. $\lim _{x \rightarrow y^{+}} \mathbf{a}_{\alpha}(x, c)=\mathbf{a}_{\alpha}(y, c)$ and $\lim _{x \rightarrow y^{-}} \mathbf{a}_{\alpha}^{\prime}(x, c)=\mathbf{a}_{\alpha}^{\prime}(y, c)$.

Proof. Again we only give the proof for $\mathbf{a}_{\alpha}$ since that for $\mathbf{a}_{\alpha}^{\prime}$ is similar. Suppose first that $y \neq 0, c, c+(\alpha / 2), 1-(\alpha / 2)$, i.e. $y$ is not an endpoint of any of the arcs $A_{i}(c)$, 
$B_{i}(c)$. Then, given any positive integer $k$ there exists $\varepsilon>0$ such that if $|x-y|<\varepsilon$ and $|n|<k$, the image pairs $r_{\alpha}^{n}(x), r_{\alpha}^{n}(y)$ lie in the same arc for each $n$. Thus $a_{\alpha}^{n}(x, c)=a_{\alpha}^{n}(y, c)$ for $|n|<k$ and

$$
\begin{aligned}
d\left(\mathbf{a}_{\alpha}(x, c), \mathbf{a}_{\alpha}(y, c)\right) & =\sum_{j=-\infty}^{\infty} \frac{\left|a_{\alpha}^{j}(x, c)-a_{\alpha}^{j}(y, c)\right|}{2^{|j|}} \\
& \leq \sum_{j=k}^{\infty} \frac{1}{2^{|j|}}+\sum_{j=-\infty}^{-k} \frac{1}{2^{|j|}}=2 \sum_{j=k}^{\infty} \frac{1}{2^{j}} \\
& =\frac{1}{2^{k-1}} \sum_{i=0}^{\infty} \frac{1}{2^{i}}=\frac{1}{2^{k-2}} .
\end{aligned}
$$

This shows that $\lim _{x \rightarrow y} \mathbf{a}_{\alpha}(x, c)=\mathbf{a}_{\alpha}(y, c)$ if $y \in \operatorname{int}\left(A_{i}(c) \cup B_{i}(c)\right), i=1,2$. If $y$ lies in an endpoint of one of the arcs then, since all points to the right of $y$ and sufficiently close to $y$ (closer than $\min (c, \alpha / 2,(1-\alpha)-c)$ ) lie in the same arc as $y$, for any positive integer $k$ we can find $\varepsilon \in(0, \min (\alpha / 2, c,(1-\alpha)-c))$ so that the symbols $a_{\alpha}^{n}(x, c)$ and $a_{\alpha}^{n}(y, c)$ agree for all $|n|<k$, as above. If $c=1-\alpha$ the proof goes through in the same fashion but with only two intervals $[0,1-\alpha)$ and $[1-\alpha, 1)$.

For $\alpha \in(0,1) \backslash \mathbb{Q}$ fixed and $c \in[0,1-\alpha)$, we define the set

$$
\Omega_{\alpha}(c)=\left\{\mathbf{a}_{\alpha}(x, c) \mid x \in S^{1}\right\} \cup\left\{\mathbf{a}_{\alpha}^{\prime}(x, c) \mid x \in S^{1}\right\} .
$$

LEMMA C. $\Omega_{\alpha}(c) \subset \Sigma$ is minimal under the shift $\sigma$ and is a Cantor set.

Proof. To show that $\Omega_{\alpha}(c)$ is minimal we must show that it is invariant under $\sigma$, closed in $\Sigma$ and that every orbit $\left\{\sigma^{k}(\mathbf{b})\right\}$ of a point $\mathbf{b} \in \Omega_{\alpha}(c)$ is dense in $\Omega_{\alpha}(c)$. Invariance follows directly from our definition of sequences (3.2) and the properties of the shift $\sigma$. To prove that $\Omega_{\alpha}(c)$ is a closed subset of $\Sigma$ it suffices to show that any infinite sequence of points of $\Omega_{\alpha}(c)$ contains a subsequence converging to a point of $\Omega_{\alpha}(c)$. Let $\left\{b\left(x_{i}, c\right)\right\}_{i=0}^{\infty}$ be such a sequence. Since $S^{1}$ is compact, the points $x_{i} \in S^{1}$ from which the symbol sequences are derived must contain a subsequence $\left\{y_{i}\right\}$ converging to a point $y \in S^{1}$ from either the right or the left. Then lemma $B$ implies that $\mathbf{a}_{\alpha}\left(y_{i}, c\right) \rightarrow \mathbf{a}_{\alpha}(y, c)$ or $\mathbf{a}_{\alpha}^{\prime}\left(y_{i}, c\right) \rightarrow \mathbf{a}_{\alpha}^{\prime}(y, c)$. Density of any orbit $\left\{\sigma^{k}\left(\mathbf{a}_{\alpha}(x, c)\right)\right\}$ or $\left\{\sigma^{k}\left(\mathbf{a}_{\alpha}^{\prime}(x, c)\right)\right\}$ in $\Omega_{\alpha}(c)$ follows directly from density of the orbit $\left\{r_{\alpha}^{k}(x)\right\}$ in $S^{1}$.

Finally, to see that $\Omega_{\alpha}(\mathrm{c})$ is a Cantor set, we note that, being a subset of $\Sigma$, it is totally disconnected, and by lemma $\mathrm{A}$ the points $\mathbf{a}_{\alpha}(x, c)\left(\right.$ resp. $\mathbf{a}_{\alpha}^{\prime}(x, c)$ ) in it are in $1: 1$ correspondence with the real numbers $x \in S^{1}$ and hence it is uncountable. Finally, since the orbit of every point $a_{\alpha}$ is dense in $\Omega_{\alpha}(c)$, each point of $\Omega_{\alpha}(c)$ is an accumulation point.

LEMMA D. There exists an uncountable set of real numbers $\mathscr{C} \subset(0,1-\alpha]$ such that if $c, c^{\prime} \in \mathscr{C}$ and $c \neq c^{\prime}$, the Cantor sets $\Omega_{\alpha}(c), \Omega_{\alpha}\left(c^{\prime}\right)$ are disjoint.

Proof. Take any pair of points $x, x^{\prime} \in S^{1}$ and suppose $c \neq(1-\alpha)-c^{\prime}$ (for otherwise the sequences $\mathbf{a}_{\alpha}(x, c)$ and $\mathbf{a}_{\alpha}\left(x^{\prime}, c^{\prime}\right)$ are identical if $\left.x^{\prime}=x+(\alpha / 2)+c^{\prime}\right)$. We claim that, if $c \neq c^{\prime}$, there exists $\varepsilon>0$ such that $d\left(\sigma^{k}\left(\mathbf{a}_{\alpha}(x, c)\right), \sigma^{k^{\prime}}\left(\mathbf{a}_{\alpha}\left(x^{\prime}, c^{\prime}\right)\right)\right)>\varepsilon$ for all $k, k^{\prime} \in \mathbb{Z}$. Indeed, suppose that $c^{\prime}-c=2 \delta>0$, so that there is a point $d \in S^{1}$ with $\delta$-neighbourhood $\left(c, c^{\prime}\right)=A_{0}\left(c^{\prime}\right) \cap B_{0}(c)$. Since the orbits $r_{\alpha}^{n}(x), r_{\alpha}^{n}\left(x^{\prime}\right)$ are dense in 
$S^{1}$, each orbit enters this $\delta$-neighbourhood infinitely many times. Let $N=N(\delta)$ denote the maximum number of iterates between any two such occurrences. Since at any such occurrence we have $r_{\alpha}^{n}(x) \in A_{0}\left(c^{\prime}\right) \cap B_{0}(c)$, the two symbol sequences differ at least once in every $N$ symbols, so that

$$
d\left(\sigma^{k}\left(\mathbf{a}_{\alpha}(x, c)\right), \sigma^{k^{\prime}}\left(\mathbf{a}_{\alpha}\left(x^{\prime}, c^{\prime}\right)\right)\right)>\frac{1}{2^{N}} \stackrel{\text { def }}{=} \varepsilon,
$$

for any pair $k, k^{\prime} \in \mathbb{Z}$, as claimed. The argument also shows that

$$
d\left(\sigma^{k}\left(\mathbf{a}_{\alpha}^{\prime}(x, c)\right), \sigma^{k^{\prime}}\left(\mathbf{a}_{\alpha}^{\prime}\left(x^{\prime}, c^{\prime}\right)\right)\right)>\varepsilon
$$

and

$$
d\left(\sigma^{k}\left(\mathbf{a}_{\alpha}(x, c)\right), \sigma^{k^{\prime}}\left(\mathbf{a}_{\alpha}^{\prime}\left(x^{\prime}, c^{\prime}\right)\right)\right)>\varepsilon .
$$

Now choose $c, c^{\prime} \in(0,1-\alpha]$ with $c^{\prime} \neq c,(1-\alpha)-c$. Defining $\Omega_{\alpha}(c)$ as above, these inequalities imply that the Cantor sets $\Omega_{\alpha}(c), \Omega_{\alpha}\left(c^{\prime}\right)$ lie a nonzero distance apart. Fixing $c$ and letting $c^{\prime}$ vary, we thus produce uncountably many disjoint Cantor sets.

LeMMA E. Let $b \in \Omega_{\alpha}(c)$. Then $R(b)=R^{-}(b)=\alpha$.

Proof. This essentially follows directly from our construction of $\Omega_{\alpha}(c)$ via the rigid rotation $r_{\alpha}(x)=x+\alpha$. (cf. [25], \$6.2). To verify that the 'symbolic' rotation number

$$
R(\mathbf{b})=\lim _{n \rightarrow \infty} \frac{1}{n} \sum_{j=0}^{n-1} b^{j}
$$

is indeed equal to $\alpha$, we consider orbits of rigid rotations $r_{p_{i} / q_{i}}$ through the rational approximants $\left\{p_{i} / q_{i}\right\}$ generated by the continued fraction expansion of $\alpha$. In particular, applying such a rotation on the circle with the irrational arcs of (3.1), the $q_{i}$-periodic orbit $\left\{r_{p_{i} / q_{i}}^{n}(x)\right\}$ consists of $q_{i}$ points spaced at intervals $1 / q_{i}$ around $S^{1}$ of which $\left[\alpha q_{i} / 2\right] \pm 1$ fall in $B_{0}(c)$ and $\left[\alpha q_{i} / 2\right] \pm 1$ in $B_{1}(c)$, where [ ] denotes the integer part. Thus the asymptotic proportion of 1's in the sequence $\mathbf{a}_{\alpha, p_{i} / q_{i}}(x, c)$ obtained from the rotation $r_{p_{i} / q_{i}}$ is bounded between $\left(\alpha q_{i}+2\right) / q_{i}$ and $\left(\alpha q_{i}-2\right) / q_{i}$ and so approaches $\alpha$ as $q_{i} \rightarrow \infty$. The same argument applies to backward orbits and thus we also find

$$
R^{-}(\mathbf{b})=\lim _{n \rightarrow \infty} \frac{1}{n} \sum_{j=0}^{n-1} b^{-j}=\alpha .
$$

3.4. Proof of theorem 3.1. Let $\mu=c /(1-\alpha) \in(0,1]$ serve as parameter for the Cantor sets $\Omega_{\alpha}(c)$. Lemma $D$ guarantees that, after deleting countably many values of $\mu$, we still have uncountably many sets $\Omega_{\alpha}(c)$ left. Picking $\alpha=\gamma N / K$, lemma E and proposition 2.7 show that each point $b \in \Omega_{\alpha}(c)$ in such a set corresponds to a point $\mathbf{x}=h^{-1}(\mathbf{b}) \in \Lambda^{N}$ with forward and backward rotation numbers $\rho(\mathbf{x})=\rho^{-}(\mathbf{x})=\gamma$. Since $h$ is a homeomorphism, the set $\Lambda_{\gamma}^{N}(\mu)=h^{-1}\left(\Omega_{\alpha}(c)\right) \subset \Lambda^{N}$ is also a Cantor set, and thus the collection $\Lambda_{\gamma}(\mu)=\bigcup_{j=0}^{N-1} f^{j}\left(\Lambda_{y}^{N}(\mu)\right)$ of its images is a Cantor set with the required properties. Letting $\mu$ range over the interval $(0,1]$ we obtain our uncountable collection.

3.5. Remarks. (1) In $\$ 3.3$, we have essentially constructed a one dimensional continuum of Cantor sets of each irrational rotation number $\gamma \in[0, K / N]$. By 
considering $n$-fold covers of the circle, rather than the twofold cover (partition into four disjoint arcs of (3.2)), one can similarly construct $n$-1-dimensional continua of Cantor sets of each irrational rotation number. This seems to be related to recent results of Mather [47] on area preserving monotone twist diffeomorphisms, although we have not attempted to prove that our sets are the same as Mather's in the case where the hypotheses of both his and our theorems are simultaneously satisfied.

(2) It is possible, at least in some cases, to give explicit algorithms for the iterative construction of irrational symbol sequences. In the case of the set $\Omega_{\alpha}(1-\alpha)$, in which the circle is partitioned into two arcs $[0,1-\alpha),[1-\alpha, 1)$, the construction directly uses the rational approximants generated by the continued fractions expansion of $\alpha$. See [65].

\section{Order preserving and non-order preserving orbits}

We now consider in more detail the construction of invariant sets analogous to $\Lambda, \Lambda^{N}$ of $\S 2.7$. In particular, we first specify conditions on the homoclinic orbit $\left\{f^{n}(\mathbf{q})\right\}_{n=-\infty}^{\infty}$ contained in the set $U \cup\left(\bigcup_{j=-(t-1)}^{k-1} f^{j}(V)\right)$ which will guarantee the existence in this set of order preserving orbits of all rotation numbers in some interval. It will turn out that most of these orbits are not invariant sets for $f^{N}$ restricted to the set $\Lambda^{N}$ constructed in $\S 2.7$, although some of the subsets of $\Lambda=\bigcup_{j=0}^{N-1} f^{j}\left(\Lambda^{N}\right)$ with well defined rotation numbers are order preserving.

We remark that a theorem of Hall [28] guarantees that any monotone twist map which possesses a $q$-periodic point of rotation number $p / q$ also possesses an order preserving (Birkhoff) $q$-periodic orbit of rotation number $p / q$. A result of Katok [38] then permits one to construct order preserving orbits of irrational rotation number by taking limits of sequences of order preserving periodic orbits. Here we wish to understand more explicitly how the orbits constructed above by 'natural' embeddings of horseshoes fail to be order preserving and so we proceed independently. Also, we do not require that $f$ be a monotone twist map. Our construction will reduce matters to the study of mappings of the circle.

4.1. Order preserving homoclinic orbits. The condition that the homoclinic orbit should not 'twist too much' is easily illustrated geometrically, and so we start with figure 3, suggested by our analysis of the Josephson equation in $\S 5$. We restrict our discussion to 1-rotary homoclinic orbits. We will require that all the $N=k+l$ iterates of the 'vertical' strips $V_{0}, V_{1}$ constructed in $\S 2.7$ project under $\Pi_{1}$ onto $S^{1}$ with the

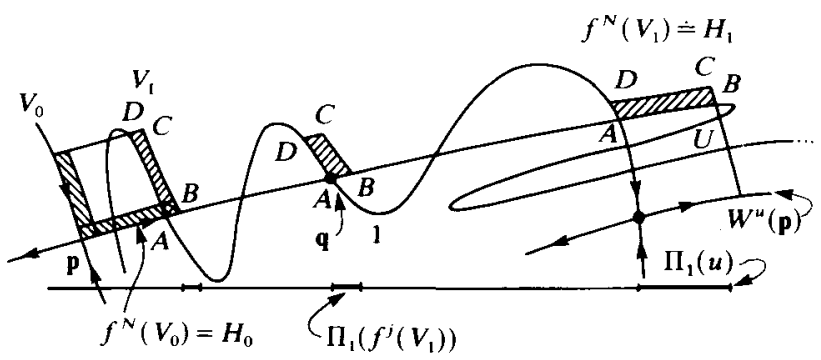

FIGURE 3. An order preserving 1-rotary transverse homoclinic orbit. 
orientations of their boundaries preserved. This clearly must happen for $\left\{f^{j}\left(V_{0}\right)\right\}_{j=0}^{N}$ since all these images are contained in $U$ and their boundaries are parallel to the local manifolds $W_{\text {loc }}^{u}(\mathbf{p}), W_{\text {loc }}^{s}(\mathbf{p})$, but care must be taken with the images $\left\{f^{j}\left(V_{1}\right)\right\}_{j=1}^{N-1}$. For example, examination of figure 2(a) will reveal that the orientations of the projected boundaries rotate through $\pi$ in that case. A homoclinic orbit $\left\{f^{n}(\mathbf{q})\right\}_{n=-\infty}^{\infty}$ for which the projections of the sets $\left\{f^{j}\left(V_{1}\right)_{j=0}^{N}\right.$ do not change orientation will be called an order preserving (1-rotary, transverse) homoclinic orbit.

The existence of such a simple homoclinic orbit will guarantee that any orbit in its neighbourhood must preserve order as it moves from $U$ around $A$ and back to $U$. Thus, if we can take care of the local behaviour in $U$, the global behaviour will take care of itself.

Henceforth in this section we assume that $\left\{f^{n}(\mathbf{q})\right\}_{n=-\infty}^{\infty}$ is an order-preserving, 1-rotary, transverse homoclinic orbit to a fixed point $\mathbf{p}$ and that we have selected an integer $N$, a neighbourhood $U$ of p and vertical strips $V_{0}, V_{1}$ such that $f^{N}\left(V_{0}\right)=H_{0}$ and $f^{N}\left(V_{1}\right)=H_{1}$ stretch 'horizontally' across $U$ and the sets $\left\{f^{j}\left(V_{1}\right)\right\}_{j=0}^{N}$ contain images of q. $U$ is supposed to be small enough ( $N$ large enough) that the maps $\left.f\right|_{U}$ and $\left.f^{N}\right|_{v_{1}}$ are uniformly hyperbolic. See figure 3 .

4.2. Induced maps. We now show that the sets $U,\left\{f^{j}\left(V_{1}\right)\right\}_{j=0}^{N}$ associated with the order preserving homoclinic orbit permit us to construct a map $\hat{g}: S^{1} \rightarrow S^{1}$ in such a way that order preserving orbits of $\hat{g}$ will correspond to order preserving orbits of $f: A \rightarrow A$. This will be done in several steps.

We choose a $(\psi, s)$ coordinate system in $U$ such that $W_{\text {loc }}^{u}(\mathbf{p})$ is the $\psi$-axis and $W_{\text {loc }}^{s}(\mathbf{p})$ the $s$-axis. Let $\left.\Pi_{\psi}\right|_{U}$ denote 'projection' along the local stable manifold onto the local unstable manifold. Let $V_{0}^{\prime}=U \cap f^{-1}(U)$ and define the induced map $g: V_{0}^{\prime} \cup V_{1} \rightarrow U$ by

$$
g(\mathbf{x})=\left\{\begin{array}{lr}
f(\mathbf{x}), & \mathbf{x} \in V_{0}^{\prime}, \\
f^{N}(\mathbf{x}), & \mathbf{x} \in V_{1} .
\end{array}\right.
$$

From the construction of $\S 2.7$ it is clear that $V_{0}^{\prime}$ and $V_{1}$ are disjoint 'vertical' strips and their images $g\left(V_{0}^{\prime}\right)=f\left(V_{0}^{\prime}\right) \stackrel{\text { def }}{=} H_{0}^{\prime}$ and $g\left(V_{1}\right)=f^{N}\left(V_{1}\right)=H_{1}$ are disjoint 'horizontal' strips which stretch across $U$ and that $g$ is uniformly hyperbolic. Note that $V_{0}$, defined in $\S 2.7$, is contained in $V_{0}^{\prime}: V_{0}^{\prime}$ is a bigger strip. The action of $g$ has the form sketched in figure $4(\mathrm{a})$.

We set up symbolic dynamics for $g$ just as we did for $\left.f^{N}\right|_{U}$ in $\$ 2.7$. Let $\Lambda^{\prime}=$ $\bigcap_{n=-\infty}^{\infty} g^{n}\left(V_{0}^{\prime} \cup V_{1}\right)$ be the (invariant) set of points $\mathrm{x} \in V_{0}^{\prime} \cup V_{1}$ which never leave $V_{0}^{\prime} \cup V_{1}$ under iteration of $g$. Assign a symbol sequence $\mathbf{a}=l(\mathbf{x})=\left\{a_{j}\right\}_{j=-\infty}^{\infty} \in \Sigma$ to each point $x \in \Lambda^{\prime}$ by the rule

$$
a_{j}(\mathbf{x})= \begin{cases}0 & \text { if } g^{j}(\mathbf{x}) \in V_{0}^{\prime}, \\ 1 & \text { if } g^{j}(\mathbf{x}) \in V_{1},\end{cases}
$$

which defines the map $l: \Lambda^{\prime} \rightarrow \Sigma$. As in (2.4) we also define the (forward) rotation number

$$
R(\mathbf{a})=\lim _{k \rightarrow \infty} \frac{1}{k} \sum_{j=0}^{k-1} a_{j}=\lim _{k \rightarrow \infty} \frac{r_{k}}{k}
$$

of a symbol sequence $a$, if the limit exists. 


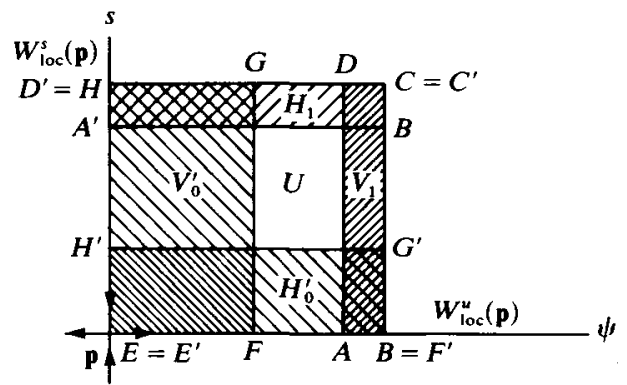

(a) the induced map $g: V_{o}^{\prime} \cup V_{1} \rightarrow U$. Points $A \cdots H$ are mapped to $A^{\prime} \cdots H^{\prime}$ by $g$.

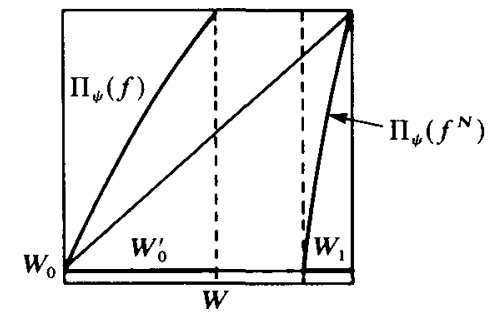

(b) the projection $\hat{g}$ of the induced map $g$ under $\Pi_{\psi}$

FIGURE 4. Induced maps of $f$.

Before we state results analogous to proposition 2.7 , we construct the one dimensional map $\hat{g}$ by projecting down the stable manifolds of $\Lambda^{\prime}$. In fact, in the local coordinate system we have chosen, the projection operator $\Pi_{\psi}$ identifies orbits in $\Lambda^{\prime}$ which have the same (forward) asymptotic behaviour. In symbolic terms, orbits with sequences whose positive entries agree are collapsed together by $\Pi_{\psi}$. Letting $W=\Pi_{\psi}(U), W_{0}=\Pi_{\psi}\left(V_{0}\right), W_{0}^{\prime}=\Pi_{\psi}\left(V_{0}^{\prime}\right)$ and $W_{1}=\Pi_{\psi}\left(V_{1}\right)$, we can represent the projected induced map $\hat{g}$ (loosely ' $\Pi_{\psi} \circ g^{\prime}$ ) as in figure $4(\mathrm{~b})$. Strictly, $\hat{g}$ is only defined on the Cantor set $\hat{\Lambda}=\Pi_{\psi}\left(\Lambda^{\prime}\right)$. Finally, identifying the endpoints of $W$, we obtain $\hat{\mathrm{g}}: S^{1} \rightarrow S^{1}$. There is a natural map $\hat{l}: \hat{\Lambda} \rightarrow \Sigma^{+}$obtained by using the recipe (4.2) for $j \geq 0$.

Returning to the two dimensional induced diffeomorphism $\mathrm{g}$, as in $\$ 2.7$ (cf. [25, $\S 5.2])$ we have:

4.3. Proposition. $\Lambda^{\prime} \subset U$ is an invariant Cantor set for $g$ and $\left.g\right|_{\Lambda^{\prime}}$ is topologically conjugate to the full shift on $\Sigma:\left.g\right|_{\Lambda^{\prime}}=l^{-1} \circ \sigma \circ l$.

Moreover, we also have:

4.4 Proposition. Suppose that $R(\mathbf{a})=\alpha \in[0,1]$ exists for $\mathbf{a} \in \Sigma$. Then the forward rotation number $\rho(\mathbf{x})$ for the point $\mathbf{x}=l^{-1}(\mathbf{a})$ exists and is given by $\gamma=$ $\alpha /[(N-1) \alpha+1]$.

Remark. Suitable choices of $\alpha \in[0,1]$ yield all $\gamma \in[0,1 / N]$. Indeed $\alpha=$ $\gamma /(1-(N-1) \gamma)$. A similar result holds for backward rotation numbers.

Proof. Consider the orbit $\left\{\mathbf{x}_{i}\right\}=\left\{g^{i}(\mathbf{x})\right\}$ of the point $\mathbf{x}=l^{-1}(\mathbf{a})$. Each ' 0 ' in a corresponds to an iterate $x_{i} \in V_{0}^{\prime}$ and each ' 1 ' in a to an iterate $x_{j} \in V_{1}$. Each of the latter 
therefore lies on an orbit which makes one full turn of $A$ in the next $N$ iterates of $f$ (just as in $\S 2,7$ ), and since $V_{0}^{\prime}=U \cap f^{-1}(U)$, each $\mathbf{x}_{i} \in V_{0}^{\prime}$ remains in $U$ for at least one iterate of $f$. (But note that such points generally do not lie in $V_{0}=$ $\bigcap_{j=0}^{N} f^{-j}(U)$ and so can leave $U$ after less than $N$ iterates.) Thus, the finite sequences with 'running average' rotation number

$$
\frac{r_{k}}{k}=\frac{1}{k} \sum_{j=0}^{k-1} a_{j}
$$

correspond to orbit segments $\left\{f^{j}(\mathbf{x})\right\}$ for points $\mathbf{x} \in U$ which describe $r_{k}$ full turns of $A$ in $N r_{k}+\left(k-r_{k}\right)$ iterations of $f$. Hence the rotation number (if it exists) will be

$$
\rho(\mathbf{x})=\lim _{k \rightarrow \infty} \frac{r_{k}}{(N-1) r_{k}+k}=\lim _{k \rightarrow \infty} \frac{r_{k} / k}{(N-1) r_{k} / k+1} .
$$

But $\lim _{k \rightarrow \infty} r_{k} / k=\alpha$ by hypothesis and thus

$$
\rho(\mathbf{x})=\frac{\alpha}{(N-1) \alpha+1}
$$

as claimed.

4.5. Order preserving orbits. Since the induced map $g: U \rightarrow U$ is conjugate to a full 2-shift, propositions 4.3 and 4.4 guarantee that orbits of all (forward) rotation numbers $\gamma \in[0,1 / N]$ for $f$ can be constructed much as in the proof of theorem 2.8. We will now show that at least one orbit of each rotation number $\gamma$ is order preserving. These orbits will generally differ from those constructed in $\$ 2.8$; in particular, for typical order preserving orbits $\left\{f^{j}(\mathbf{x})\right\}$ the set $\left\{f^{j}(\mathbf{x})\right\}_{j=-\infty}^{\infty} \cap U$ will not be invariant for $f^{N}$ (see $\S 4.7$, below).

Consider the one dimensional map $\hat{g}: \hat{\Lambda} \rightarrow \hat{\Lambda}\left(\hat{\Lambda} \subset S^{1}\right)$ and the associated symbolic dynamics induced by $\hat{l}: \hat{\Lambda} \rightarrow \Sigma^{+}$described in $\S 4$.2. Suppose that the induced map $g: \Lambda^{\prime} \rightarrow \Lambda^{\prime}$ possesses an orbit which is badly ordered, i.e. there exists $x \in \Lambda^{\prime}$ such that $\Pi_{\psi}(\mathbf{x})<\Pi_{\psi}(g(\mathbf{x}))$ but $\Pi_{\psi}\left(g^{j}(\mathbf{x})\right)>\Pi_{\psi}\left(g^{j+1}(\mathbf{x})\right)$ for some $j>0$. Then it is obvious from the definition of $\hat{g}$ that $y=\Pi_{\psi}(\mathbf{x}) \in \hat{\Lambda}$ has an orbit which is badly ordered. Thus if $y \in \hat{\Lambda}$ has an order preserving orbit under $\hat{g}$, then there must exist $\mathbf{x} \in \Pi_{\psi}^{-1}(y)$ whose orbit under $g$ is order preserving, for since $y$ has an order preserving orbit, it cannot be the image of a point with a badly ordered orbit.

In view of the above remark, it is sufficient to construct order preserving orbits for the one dimensional map $\hat{g}$, and we will do this by constructing 'correctly ordered' symbol sequences, which requires the following elementary result:

LEMMA F: Let $\phi_{1}, \phi_{2} \in \hat{\Lambda}=\Pi_{\psi}\left(\Lambda^{\prime}\right)$ and let $\hat{l}\left(\phi_{1}\right), \hat{l}\left(\phi_{2}\right) \in \Sigma^{+}$denote the semi-infinite symbol sequences associated with these points. Then $\phi_{1}<\phi_{2} \Leftrightarrow \hat{l}\left(\phi_{1}\right)<\hat{l}\left(\phi_{2}\right)$, where denotes lexicographic ordering.

Proof. Suppose $\phi_{1}<\phi_{2}$ and $\phi_{1}, \phi_{2}$ lie in the same subinterval $W_{0}^{\prime}$ or $W_{1}$, for if they do not then $\left(\hat{l}\left(\phi_{1}\right)\right)_{1} \neq\left(\hat{l}\left(\phi_{2}\right)\right)_{1}$ and there is nothing to prove. Since $\left.g\right|_{v_{0}^{\prime} \cup v_{1}}$ is uniformly hyperbolic, we have $g^{\prime} \geq c>1$ on $W_{0}^{\prime} \cup W_{1}$. Thus there exists an integer $j \geq 0$ such that $\hat{g}^{j}\left(\phi_{1}\right), \hat{g}^{j}\left(\phi_{2}\right)$ lie in different subintervals; let $J$ be the smallest such, so that both members of the pair $\hat{g}^{i}\left(\phi_{1}\right), \hat{g}^{i}\left(\phi_{2}\right)$ lie in the same subinterval ( $W_{0}^{\prime}$ or $W_{1}$ ) for each $0 \leq i<J$. Thus $\hat{g}^{J-1}\left(\phi_{1}\right)<\hat{g}^{J-1}\left(\phi_{2}\right)$ and $\hat{g}^{J}\left(\phi_{1}\right) \in W_{0}^{\prime}, \hat{g}^{J}\left(\phi_{2}\right) \in W_{1}$ (cf. 
figure $4(\mathrm{~b}))$ and so $\left(\hat{l}\left(\phi_{1}\right)\right)_{i}=\left(\hat{l}\left(\phi_{2}\right)\right)_{i}$ for $0 \leq i<J$ and $\left(\hat{l}\left(\phi_{1}\right)\right)_{J}=0,\left(\hat{l}\left(\phi_{2}\right)\right)_{J}=1$, implying that $\hat{l}\left(\phi_{1}\right) \cup \hat{l}\left(\phi_{2}\right)$ as required.

Now suppose $\hat{l}\left(\phi_{1}\right) \downarrow \hat{l}\left(\phi_{2}\right)$ and that the sequences differ first at the $J$ th symbol: $\left(\hat{l}\left(\phi_{1}\right)\right)_{J}=0,\left(\hat{l}\left(\phi_{2}\right)\right)_{J}=1$. This implies that $\hat{g}^{J}\left(\phi_{1}\right) \in W_{0}^{\prime}, \hat{g}^{J}\left(\phi_{2}\right) \in W_{1}$ and hence that $\hat{g}^{J-1}\left(\phi_{1}\right)<\hat{g}^{J-1}\left(\phi_{2}\right)$ and both points lie in the same subinterval. Therefore $\hat{\mathrm{g}}^{i}\left(\phi_{1}\right)<\hat{\mathrm{g}}^{i}\left(\phi_{2}\right)$ for all $0 \leq i<J$ and in particular $\phi_{1}<\phi_{2}$.

We recall from $\S 3$ the construction of the Cantor set $\Omega_{\alpha}(c)$. In particular, when $c=1-\alpha$, the partition of the circle degenerates into two arcs $A=[0,1-\alpha), B=$ $[1-\alpha, 1)$. We will show that the image of the Cantor set $\Omega_{\alpha}(1-\alpha)$ under $\hat{l}^{-1}$ is a well ordered subset of $\hat{\Lambda}$ and, as a consequence, also well ordered for $f$.

LeMma G. Let $\mathbf{a} \in \Omega_{\alpha}(1-\alpha), \alpha \in[0,1]$. Then $y=\hat{l}^{-1}(\mathbf{a}) \in \hat{\Lambda}$ has an order preserving orbit under $\hat{g}$ with $\rho(y)=\alpha$. Hence there exists $\mathbf{x} \in \Pi_{\psi}^{-1}(y)$ whose orbit is well-ordered under $\mathrm{g}$.

Proof. That a and its shifts are properly ordered is essentially obvious from the construction of $\Omega_{\alpha}(1-\alpha)$ of $\S 3.3$. All that remains to be shown is that $a=\hat{l}(y)$ for some $y \in \hat{\Lambda}$, i.e. that a actually represents the dynamics under $\hat{g}$ of some $y \in \hat{\Lambda}$. But this follows from proposition 4.3 and the definitions of $\hat{g}$ and $\hat{\Lambda}$. The last statement follows from the remarks preceding lemma $F$.

To conclude the construction of order preserving orbits for $f$ we need:

LEMMA H. Let $\mathbf{x} \in \Lambda^{\prime} \subset U$. If the orbit of $\mathbf{x}$ is order preserving for $g: U \rightarrow U$ then it is also order preserving for $f: A \rightarrow A$.

Proof. Since $\mathrm{g}=f$ on $V_{0}^{\prime} \subset U$ we need only consider the $f$-orbits of points $\mathrm{x} \in V_{1}$. But our assumption of an order preserving homoclinic orbit $(\$ 4.1)$ implies that the images $f^{j}(\mathbf{x}), f^{j}(\mathbf{y}), 0<j<N$, of any pair of points $\mathbf{x}, \mathbf{y} \in V_{1}$ retain the same order as these points are mapped around $A$ by $f$. Thus $f^{N}(\mathbf{x}), f^{N}(\mathbf{y})$ land in $U$ in the same order in which they left $U$ and order is preserved for $f$ as well as for $g=f^{N} \mid V_{1}$.

We have now proved the following.

4.6. Proposition. If $f: A \rightarrow A$ has an order preserving homoclinic orbit near which there is an orbit of rotation number $\gamma$ then $f$ has an order preserving orbit of rotation number $\gamma$.

Remark. The conclusion of this result is similar to that of Hall [28] and Boyland and Hall [13]. In fact one can probably weaken our hypotheses, since in topologically 'reasonable' situations, the existence of a non-order preserving homoclinic orbit seems to imply the existence of an order preserving one.

4.7. Some examples. Here we use the algorithm described in $\$ 3.3$ and the results of $\S \S 4.1-6$ to construct some order preserving orbits for $f$. We also demonstrate that orbits constructed from the 'natural' embedding of the horseshoe for $f^{N}$ described in $\S 2$ are not generally order preserving.

We briefly summarize the procedure. To construct an order preserving invariant set for $f$ of rotation number $\gamma \in[0,1 / N]$ we let $\alpha=\gamma /(1-(N-1) \gamma)$ and construct 
$\mathbf{a}(x, 1-\alpha)$, where $x \in[0,1]$ is arbitrary, by eq. (3.2). We interpret $\mathbf{a}(x, 1-\alpha)$ as arising from the symbolic dynamics induced by the projected induced map $\hat{\mathrm{g}}$. Lexicographical ordering of $a(x, 1-\alpha)$ and its shifts gives the ordering of the iterates $\hat{g}^{k}(y)$ of the corresponding point $y=\hat{l}^{-1}(\mathbf{a}(x, 1-\alpha))$ in $W=\Pi_{\psi}(U)$. Choosing a particular $\mathbf{x} \in \Pi_{\psi}^{-1}(y)$ gives a point whose orbit under the induced map $g$ is order preserving and has rotation numbers $\alpha$ for $g$ and $\gamma$ for $f$. The actual invariant set for $f$ is then obtained by following the $N-1$ images under $f$ of those points $g^{k}(\mathbf{x})$ which lie in $V_{1}$ (noting that $g=f$ on $V_{0}^{\prime}$ ).

Example (a). Let $N=3$ and $\gamma=\frac{2}{9}$. Then $\alpha=\frac{2}{9} /\left(1-(3-1) \cdot \frac{2}{9}\right)=\frac{2}{5}$. Letting $x=\frac{1}{4}$ we find $\mathbf{a}\left(\frac{1}{4}, \frac{3}{5}\right)={ }^{-}(01001)^{+}$. If we let $\mathbf{c}^{j}=\sigma^{j}\left(\mathbf{a}\left(\frac{1}{4}, \frac{3}{5}\right)\right), 0 \leq j \leq 4$, then the points $\phi_{j}=\hat{l}^{-1}\left(\mathbf{c}^{j}\right)$ are ordered in $W=\Pi_{\psi}(U)$ as indicated in figure $5(\mathrm{a})$, where $\phi_{j+1}=\hat{g}\left(\phi_{j}\right), 0 \leq j \leq 3$, $\phi_{0}=\hat{g}\left(\phi_{4}\right)$.

The projection of the corresponding orbit for $f$ is shown in figure 5(b).

Example (b). Let $N=2$ and $\gamma=(\sqrt{5}-1) /(\sqrt{5}+1)$. Then $\alpha=(\sqrt{5}-1) / 2$, the golden mean.

Again taking $x=\frac{1}{4}$, we find that the first 20 elements of $\mathbf{a}(x, 1-\alpha)=\mathbf{a}\left(\frac{1}{4},(3-\sqrt{5}) / 2\right)$ are 01101011011010110101 . There is enough information here to order lexicographically $\sigma^{j}(\mathbf{a}(x, 1-\alpha)) \stackrel{\text { def }}{=} \mathbf{c}^{j}$ for $0 \leq j \leq 9$, and hence to determine the relative position in $W$ of $\phi_{j}=\hat{l}^{-1}\left(\sigma^{j}(\mathbf{a}(x, 1-\alpha))\right)$ for $0 \leq j \leq 9$. See figures $5(\mathrm{c})$, (d).

\begin{tabular}{lcc}
$j$ & $\mathbf{c}^{j}($ first 11 symbols $)$ & Lexicographic postion \\
\hline 0 & 01101011011 & 3 \\
1 & 11010110110 & 9 \\
2 & 10101101101 & 5 \\
3 & 01011011010 & 1 \\
4 & 10110110101 & 7 \\
5 & 01101101011 & 4 \\
6 & 11011010110 & 10 \\
7 & 10110101101 & 6 \\
8 & 01101011010 & 2 \\
9 & 11010110101 & 8
\end{tabular}

Example (c). Let $N=3$ and $\gamma=\frac{2}{5}$, so that $\alpha=\frac{2}{15}$. We will construct two disjoint orbits of rotation number $\frac{2}{5}$ for $\left.f^{3}\right|_{U}$ using the embeddings of $\Lambda^{N}$ and $\Lambda \subset A$ of $\S 2.7-8$. Consider the pre-images $\mathbf{x}=h^{-1}(\mathbf{a}), y=h^{-1}(\mathbf{b})$ for $\mathbf{a}={ }^{-}(00101)^{+}, \mathbf{b}=$ ${ }^{-}(00011)^{+} \in \Sigma$. We observe that $a(0,0.6)=(00101)^{+}$, using the construction of $\S 3.3$, and that $(00011)^{+} \neq \mathbf{a}(x, 0.6)$ for any $x \in[0,1]$. The reader can check that the orbit $\left\{f^{3 j}(\mathbf{x})\right\}_{j=-\infty}^{\infty}$ is order preserving for $\left.f^{3}\right|_{U}$, while $\left\{f^{3 j}(\mathbf{y})\right\}_{j=-\infty}^{\infty}$ is not. Moreover, neither $\left\{f^{j}(\mathbf{y})\right\}_{j=-\infty}^{\infty}$ nor $\left\{f^{j}(\mathbf{x})\right\}_{j=-\infty}^{\infty}$ are order preserving for $f: A \rightarrow A$. See figures 5(e),(f). For $\mathrm{x}=h^{-1}$ (a) this occurs despite the fact that a can be generated using the same symbolic recipe that led to order preserving orbits in examples (a) and (b). Thus the 'natural' embedding of the horseshoes of $\S 2$ does not, in general, yield order preserving orbits, either for $f$ or $f^{N}$. 


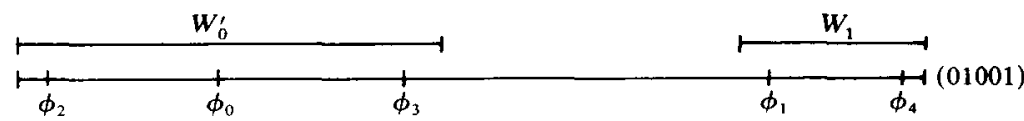

(a) An order preserving orbit of rotation number $\alpha=\frac{2}{5}$ for $\hat{g}$. The indices reflect the order of visitation under the action of $\hat{\mathrm{g}}$, starting at $\phi_{0}$.

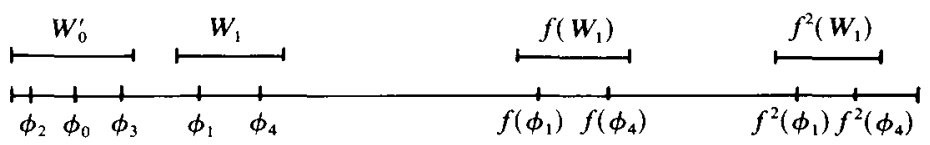

(b) The projection of the order preserving orbit for $f$ obtained from (a). Note that $\phi_{2}=f^{3}\left(\phi_{1}\right), \phi_{0}=f^{3}\left(\phi_{4}\right)$ and that the rotation number for this orbit is $\gamma=\frac{2}{9}$.

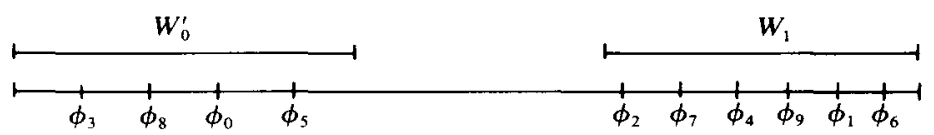

(c) The first 9 iterates under $\hat{g}$ of a point $\phi_{0}$ belonging to an order preserving Cantor set of rotation number $\alpha=(\sqrt{5}-1) / 2$.

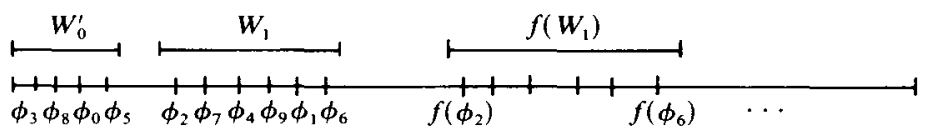

(d) The projection of part of the order preserving Cantor set of rotation number $\gamma=(\sqrt{5}-1) /(\sqrt{5}+1)$ for $f$.

Figure 5(i). Order preserving orbits for $\hat{g}$ and $f$.

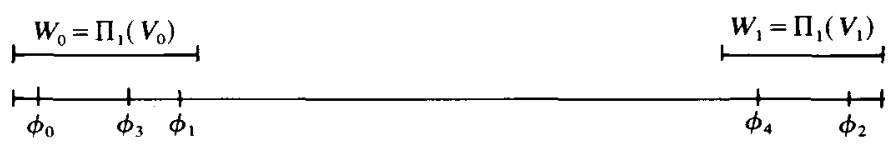

(e) An order preserving orbit for $\left.f^{3}\right|_{U}(00101)$.

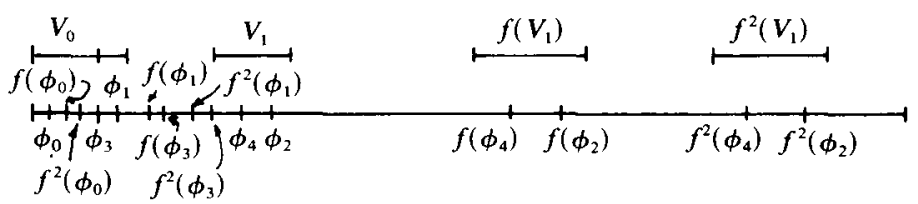

(f) The same orbit as in (e) is not order preserving for $f$. Note that $f^{3}\left(\phi_{i}\right)=\phi_{i+1}$.

FIGURE 5(ii). Non order preserving orbits for $f$.

\section{Application to the Josephson junction equation}

We now turn to the single degree of freedom Josephson equation

$$
\left.\begin{array}{l}
\dot{\phi}=r \\
\dot{r}=-\sin \phi+\nu-\delta r+\beta \cos \omega t
\end{array}\right\} ; \quad(\phi, r, t) \in A \times S^{1},
$$

and the associate Poincaré map $P_{\varepsilon}=P_{\nu, \delta, \beta, \omega}: A \rightarrow A$ defined in $\S 1$. We first show 
that (5.1) has a Birkhoff attracting set by finding a trapping region for $P$. We then use the Melnikov [49] perturbation method (cf. [23], [25], \$\$ 4.5-7] and [22]) to prove that the attracting set contains transverse homoclinic orbits. In fact the perturbation theory shows that one of these orbits is order preserving and 1-rotary, so that the results of $\S \S 2-4$ above can be used to obtain detailed information on subsets of orbits within the attracting set. We also estimate the integer $N$ of $\S 2.7$ in terms of the parameters $\nu, \delta, \beta, \omega$ of (5.1) and end the section with some conjectures and examples of 'pathological' Birkhoff attracting sets.

5.1. Proposition. Let $B=\left\{(\phi, r) \in A \mid r \in\left[\Delta_{1}, \Delta_{2}\right]\right\}$. If $\Delta_{1}<(\nu-\beta-1) / \delta, \Delta_{2}>$ $(\nu+\beta+1) / \delta$, and $\nu, \beta \geq 0, \delta>0$, then the set $\mathscr{A}=\bigcap_{n=0}^{\infty} P_{\varepsilon}^{n}(B)$ is an attracting set for the Josephson Poincaré map. A has zero Lesbesgue measure and hence empty interior.

Proof. We show that the (time dependent) vector field of (5.1) is directed inwards on the boundary components $r=\Delta_{1,2}$ of $B$ for all $t$ :

$$
\begin{aligned}
r=\Delta_{2}: \dot{r} & =-\sin \phi+\nu-\delta \Delta_{2}+\beta \cos \omega t \\
& \leq-\delta \Delta_{2}+1+\nu+\beta \\
& \leq 0, \quad \text { if } \Delta_{2}>(\nu+\beta+1) / \delta ; \\
r=\Delta_{1}: \dot{r} & =-\sin \phi+\nu-\delta \Delta_{1}+\beta \cos \omega t \\
& \geq-\delta \Delta_{1}-1+\nu-\beta \\
& \geq 0 \quad \text { if } \Delta_{1}<(\nu-\beta-1) / \delta .
\end{aligned}
$$

Thus $B$ is a trapping region for $P_{\varepsilon}: A \rightarrow A$ and $\mathscr{A}$ is its associated attracting set (cf. $\$ 2.5$ ).

To see that $\mathscr{A}$ has zero area, we note that the divergence of the vector field $(5.1)$

$$
\frac{\partial}{\partial \phi}(\dot{\phi})+\frac{\partial}{\partial r}(\dot{r})=-\delta
$$

is constant and strictly negative, and hence, via standard linear analysis, that $\operatorname{det}\left(D P_{\varepsilon}\right)=e^{-\delta \cdot 2 \pi / \omega} \stackrel{\text { def }}{=} \zeta<1$ for $\delta>0$. Thus the areas of the images $P_{\varepsilon}^{n}(B)$ decrease geometrically at the rate $\zeta^{n}$ and since $\zeta^{n} \rightarrow 0$ as $n \rightarrow \infty, \mathscr{A}$ has zero area.

5.2. Melnikov's Method. We recall the basic features of the Melnikov perturbation method for two dimensional time periodic vector fields of the form

$$
\dot{\mathbf{x}}=\mathbf{f}(\mathbf{x})+\varepsilon \mathbf{g}(\mathbf{x}, t ; \boldsymbol{\mu}) ; \quad \mathbf{x}=\left(\begin{array}{l}
u \\
v
\end{array}\right) \in M^{2}\left(=S^{1} \times \mathbb{R} \text { here }\right)
$$

with $0 \leq \varepsilon \ll 1$ and $g T$-periodic in $t$ and also depending on parameters $\mu \in \mathbb{R}^{k}$. We suppose that

$$
\mathbf{f}=\left(\begin{array}{c}
\partial F / \partial v \\
-\partial F / \partial u
\end{array}\right)
$$

is Hamiltonian and has a homoclinic orbit $\gamma^{\circ}(t)$ to a hyperbolic saddle point $p$ and moreover that $\gamma^{0}(t)$ is the limit of a continuous family $\gamma^{h}(t)$ of periodic orbits whose periods $\tau(h) \rightarrow \infty$ monotonically as $h \rightarrow h_{0}$. Here $h$ can be taken to be the Hamiltonian energy $F\left(\gamma^{h}\right)$ of the orbit and $h_{0}$ is the energy $F\left(\gamma^{0}\right)$ of the homoclinic orbit. We also assume that $d T / d h>0$, at least for $h$ near $h_{0}$. 
We define the $\wedge$-product of 2-vectors $\mathbf{a}, \mathbf{b}$ as $\mathbf{a} \wedge \mathbf{b}=a_{1} b_{2}-a_{2} b_{1}$. The Melnikov functions are then defined for the homoclinic loop $\gamma^{0}$ and for $T$-resonant members $\gamma^{h(m, n)}$ of period $\tau(h(m, n))=m T / n,(m, n)=1$, of the periodic family $\left\{\gamma^{h}\right\}$ as follows:

$$
\begin{aligned}
M^{\infty}\left(t_{0} ; \boldsymbol{\mu}\right) & =\int_{-\infty}^{\infty}(\mathbf{f} \wedge \mathbf{g})\left(\gamma^{0}(t), t+t_{0} ; \boldsymbol{\mu}\right) d t \\
M^{m / n}\left(t_{0} ; \boldsymbol{\mu}\right) & =\int_{0}^{m T}(\mathbf{f} \wedge \mathrm{g})\left(\gamma^{h(m, n)}(t), t+t_{0} ; \boldsymbol{\mu}\right) d t
\end{aligned}
$$

The basic results are as follows:

5.3. THEOREM. If $M^{\infty}$ (resp. $M^{m / n}$ ) has a simple zero as a function of $t_{0}$ for fixed $\boldsymbol{\mu}$ then, for $\varepsilon \neq 0$ sufficiently small, the Poincare map of (5.2) has a hyperbolic fixed point $\mathbf{p}_{\varepsilon}=\mathbf{p}+O(\varepsilon)$ whose stable and unstable manifolds intersect transversely (resp. the Poincaré map of (5.2) has an isolated m-periodic subharmonic orbit).

For a proof see $\$ \S 4.5-6$ (theorems 4.5 .3 and 4.6.2) of [25]. Bifurcation theorems with hypotheses involving degenerate (quadratic) zeros of $\boldsymbol{M}$ are also available, but they will not be used explicitly in this paper. However, the fact that regular perturbation expansions are used implies that the perturbed homoclinic and periodic orbits inherit important aspects of the geometric structure of the unperturbed orbits $\mathbf{x}_{0}(t)$ of (5.2) with $\varepsilon=0$. This allows us to draw conclusions on order preserving orbits, as in the following subsections.

5.4. The Josephson equation. To put (5.1) into the form (5.2) we assume that the damping $(\delta)$, constant $(\nu)$ and periodic $(\beta)$ driving amplitudes are uniformly small and write $\delta=\varepsilon \bar{\delta}, \nu=\varepsilon \bar{\nu}, \beta=\varepsilon \bar{\beta}$ to obtain

$$
\begin{aligned}
& \dot{\phi}=r \\
& \dot{r}=-\sin \phi+\varepsilon(\bar{\nu}-\bar{\delta} r+\bar{\beta} \cos \omega t) .
\end{aligned}
$$

Thus the unperturbed system has Hamiltonian

$$
F(\phi, r)=\frac{r^{2}}{2}+(1-\cos \phi)=h
$$

with the well known pendulum phase portrait containing two seperatrix or homoclinic loop solutions (cf. figure 8(a)):

$$
\boldsymbol{\gamma}^{0}(t)=( \pm 2 \arctan (\sinh t), \pm 2 \operatorname{sech} t)
$$

with energy $h^{0}=2$, and a family of rotary periodic solutions

$$
\gamma^{h}(t)=\left( \pm 2 \arcsin \left[\operatorname{sn}\left(\sqrt{\frac{h}{2}} t \mid \frac{2}{h}\right)\right], \pm \sqrt{2 h} \mathrm{dn}\left(\sqrt{\frac{h}{2}} t \mid \frac{2}{h}\right)\right),
$$

with energies $h \geq 2$. Here sn and $\mathrm{dn}$ are the Jacobi elliptic functions. There are also oscillatory periodic solutions with energies $h \in(0,2)$, but we will not use these in the computations below. The periods of the rotary family (5.8) satisfy

$$
\tau(h)=2 \sqrt{\frac{2}{h}} K\left(\frac{2}{h}\right)
$$

where $K$ is the complete elliptic integral of the first kind. 
We shall concentrate on a particular parameter region in which $\nu, \beta, \delta$ are all positive and the time independent perturbations $\nu$ and $-\delta r$ balance near the upper $(+)$ unperturbed homoclinic loop $\gamma^{0}(t)$. We will -find embedded horseshoes and complicated rotational behaviour in the neighbourhood of this orbit. Let $P_{0}$ denote the Poincaré map of the unperturbed $(\varepsilon=0)$ Hamiltonian system of $(5.1)$ and $P_{\varepsilon}$ or $\boldsymbol{P}_{\nu, \beta, \delta, \omega}$ denote the perturbed map.

Before proceeding, we note several earlier studies of the Josephson system (5.1) and relatives of it. Levi et al. [43] and Sanders and Cushman [60] consider the autonomous $(\beta=0)$ case and Belykh et al. [6], Abidi and Chua [1], Odyniec and Chua [53] and Salam and Sastry [59] consider the periodically forced case. Some of the Melnikov computations used in the proof of theorem 5.6 appeared in these earlier papers. There are also several numerical studies, including those of Huberman et al. [35], Jensen et al. [37], and Bohr et al. [11], the latter two addressing the question of rotation numberrs and the conjectural reduction of the Poincare map to a circle map. As our results show, this is clearly not generally globally possible, although individual order preserving invariant subsets can be projected in a $1: 1$ fashion onto a circle.

We start with an elementary remark on the unperturbed Poincaré map.

5.5. Proposition. All orbits of the unperturbed Poincaré map $P_{0}$ of (5.1) which lie on level sets $F^{-1}(h), h \geq 2$, are order preserving. Moreover, the unperturbed homoclinic orbits are 1-rotary.

Proof. From (5.6), each $h>2$ level set of $F$ is a simple closed curve encircling the phase cylinder $S^{1} \times \mathbb{R}$ in the $\phi$ direction. Moreover, the curves are graphs over $\phi$. Such a curve is invariant for the $\varepsilon=0$ flow and hence also an invariant manifold for $P_{0}$, since $P_{0}$ is obtained simply by integrating the unperturbed flow for time $T=2 \pi / \omega$. The map $\left.P_{0}\right|_{h=\text { const. }}$ restricted to any such manifold is a diffeomorphism of the circle and hence all orbits are automatically order preserving: in a suitable (action angle) coordinate system each such restricted map is simply a rigid rotation. Letting $h \rightarrow 2^{+}$and limiting on the homoclinic orbit $\gamma^{0}(t)$ we still have an invariant manifold $F^{-1}(2) \cup\{\mathbf{p}=( \pm \pi, 0)\}$ homotopic to the circles $F^{-1}(h), h>2$, and thus the homoclinic orbit is also order preserving. It is also clearly 1-rotary since $\phi(t)$ changes by $2 \pi$ (from $-\pi$ to $\pi$ ) as $t$ runs from $-\infty$ to $+\infty$ (eqn. (5.7)).

We now give our main perturbation results.

5.6. ThEOREM. Assume that $\nu, \beta, \delta>0$ are sufficiently small and all of the same order. If $\beta>\beta_{\infty}=|(\pi \nu-4 \delta) / \pi \operatorname{sech}(\pi \omega / 2)|$ then the Poincare map $P_{\varepsilon}$ for (5.1) has an order preserving, 1-rotary, transverse homoclinic orbit to a hyperbolic saddle point $\mathbf{p}_{\varepsilon}=(\pi, 0)+\mathcal{O}(\varepsilon)$. Furthermore, if

$$
\beta>\beta_{m}=\left|\frac{\pi \nu-4 \delta \sqrt{(h / 2)} E(2 / h)}{\pi \operatorname{sech}\left[\omega \sqrt{(2 / h)} K^{\prime}(2 / h)\right]}\right|,
$$

where $h=h(m)$ solves $\sqrt{(2 / h)} K(2 / h)=\pi m / \omega$, then the Poincaré map $P_{\varepsilon}$ for $(5.1)$ has precisely two order preserving m-periodic orbits of rotation number $1 / \mathrm{m}$.

Remark. Here $K$ and $E$ are the complete elliptic integrals of the first and second 
kinds respectively and $K^{\prime}$ is the complementary complete elliptic integral of the first kind. See [14].

Proof. We compute the Melnikov functions for (5.5) and use theorem 5.3. Starting with the homoclinic orbit, we have

$$
\begin{aligned}
M^{\infty}\left(t_{0} ; \bar{\nu}, \bar{\beta}, \bar{\delta}, \omega\right) & =\int_{-\infty}^{\infty}(r(t),-\sin \phi(t)) \wedge\left(0, \bar{\nu}-\bar{\delta} r(t)+\bar{\beta} \cos \omega\left(t+t_{0}\right)\right) d t \\
& =\int_{-\infty}^{\infty}\left(\bar{\nu} r(t)-\bar{\delta} r^{2}(t)+\bar{\beta} r(t) \cos \omega\left(t+t_{0}\right)\right) d t
\end{aligned}
$$

Using $r(t) d t=d \phi$ and $F(\phi, r)=2$ in (5.6) on the homoclinic orbit $\gamma^{0},(5.10)$ can be rewritten

$$
\begin{aligned}
M^{\infty}\left(t_{0} ; \bar{\nu}, \bar{\beta}, \bar{\delta}, \omega\right) & =\int_{-\pi}^{\pi}[\bar{\nu}-\bar{\delta} \sqrt{2(1+\cos \phi)}] d \phi+2 \bar{\beta} \int_{-\infty}^{\infty} \operatorname{sech} t \cos \omega\left(t+t_{0}\right) d t \\
& =\int_{-\pi}^{\pi}\left[\bar{\nu}-2 \bar{\delta} \cos \frac{\phi}{2}\right] d \phi+2 \bar{\beta} \int_{-\infty}^{\infty}[\operatorname{sech} t \cos \omega t] d t \cos \omega t_{0},
\end{aligned}
$$

where we have substituted the unperturbed ' $t$ ' solution (5.7), expanded using trigonometrical identities, and used the fact that the integral of the odd function $\operatorname{sech} t \sin \omega t \equiv 0$. Finally, (5.11) yields

$$
M^{\infty}\left(t_{0} ; \bar{\nu}, \bar{\beta}, \bar{\delta}, \bar{\omega}\right)=2 \pi \bar{\nu}-8 \bar{\delta}+2 \pi \beta \operatorname{sech}(\pi \omega / 2) \cos \omega t_{0} .
$$

Now (5.12) has precisely two simple zeros in each $T=2 \pi / \omega t_{0}$-interval if $\bar{\beta}>$ $|(2 \pi \bar{\nu}-8 \bar{\delta}) / 2 \pi \operatorname{sech}(\pi \omega / 2)|$, or, returning to $\nu, \beta, \delta$ by multiplying $\bar{\nu}, \bar{\beta}, \bar{\delta}$ by $\varepsilon$, if

$$
\beta>\beta_{\infty} \stackrel{\text { def }}{=}\left|\frac{\pi \nu-4 \delta}{\pi \operatorname{sech}(\pi \omega / 2)}\right| .
$$

Theorem 5.3 then implies that, for $\nu, \beta, \delta$ all of $O(\varepsilon)$ and $\varepsilon \neq 0$ sufficiently small, the unperturbed ' $t$ ' homoclinic loop $\gamma^{0}(t)$ to the saddle $p=(\pi, 0)$ breaks to give transverse homoclinic orbits to the perturbed saddle $\mathbf{p}_{\varepsilon}=\mathbf{p}+\mathcal{O}(\varepsilon)$. We remark that, for $\nu, \delta>0$ and $\beta<|(\pi \nu+4 \delta) / \pi \operatorname{sech}(\pi \omega / 2)|$, the lower $(-)$ homoclinic loop is broken completely and no homoclinic orbits with $r \leq 0$ survive. To see this, replace $r(t)$ by $-2 \operatorname{sech} t$ in (5.10) and (5.11) and recompute to obtain

$$
M^{\infty}\left(t_{0} ; \bar{\nu}, \bar{\beta}, \bar{\delta}, \omega\right)^{-}=-2 \pi \bar{\nu}-8 \bar{\delta}-2 \pi \bar{\beta} \operatorname{sech}(\pi \omega / 2) \cos t_{0} .
$$

To see that the transverse homoclinic points corresponding to the zeros of $M^{\infty}$ are 1-rotary, we must examine the perturbation method more closely. As Greenspan and Holmes [23] show (cf. [25, §§ 4.5-6]), $\varepsilon$-power series expansions are valid in the intervals $t \in\left(-\infty, t_{0}\right]$ if $\mathbf{x}_{\varepsilon} \in W^{u}(\mathbf{p o})$ and $t \in\left[t_{0}, \infty\right)$ if $\mathbf{x}_{\varepsilon} \in W^{s}(\mathbf{p o})$, where $W^{u}, W^{s}$ denote the two dimensional unstable and stable manifolds of the periodic orbit po $(t)=\mathbf{p}+O(\varepsilon)$ of the suspended flow (1.3). This just expresses the fact that perturbed homoclinic orbits must remain close to their unperturbed 'parents' for all $t \in(-\infty, \infty)$. Thus any such orbit corresponding to a zero of $M^{\infty}$ must remain in an $\varepsilon$-neighbourhood of the 1-rotary orbit $\gamma^{\circ}(t)$ and consequently must be 1-rotary itself for small $\varepsilon$. 
We now show that one member of the pair of zeros of $M^{\infty}$ in $t_{0} \in[0,2 \pi / \omega)$ corresponds to an order preserving homoclinic orbit (its partner is not order preserving, but rather twists neighbouring points through $180^{\circ}$ as they move once around $A$, cf. figure 2(a)). As shown in $[25, \S 4.5]$, the geometry associated with the Melnikov measure of homoclinic splitting is as shown in figure 6. If the oriented distance function $d\left(t_{0}\right)>0, W^{u}\left(\mathbf{p}_{\varepsilon}\right)$ lies above $W^{s}\left(\mathbf{p}_{\varepsilon}\right)$ at $\mathbf{x}(0)$ on the section $\Sigma_{t_{0}}$, while if $d\left(t_{0}\right)<0, W^{u}\left(\mathbf{p}_{\varepsilon}\right)$ lies below $W^{s}\left(\mathbf{p}_{\varepsilon}\right)$ : figure 6(a). Thus the homoclinic orbits corresponding to neighbouring zeros of $M^{\infty}$ at $t_{0}=\tau_{1}, \tau_{2} \in[0,2 \pi / \omega)$ involve opposite orientations of the tangent spaces of the intersecting manifolds. Figure 6(b) (cf. figures 2(a) and 3) should make it clear that one (and only one) of these orbits is order preserving in the sense of $\S 4.1$.
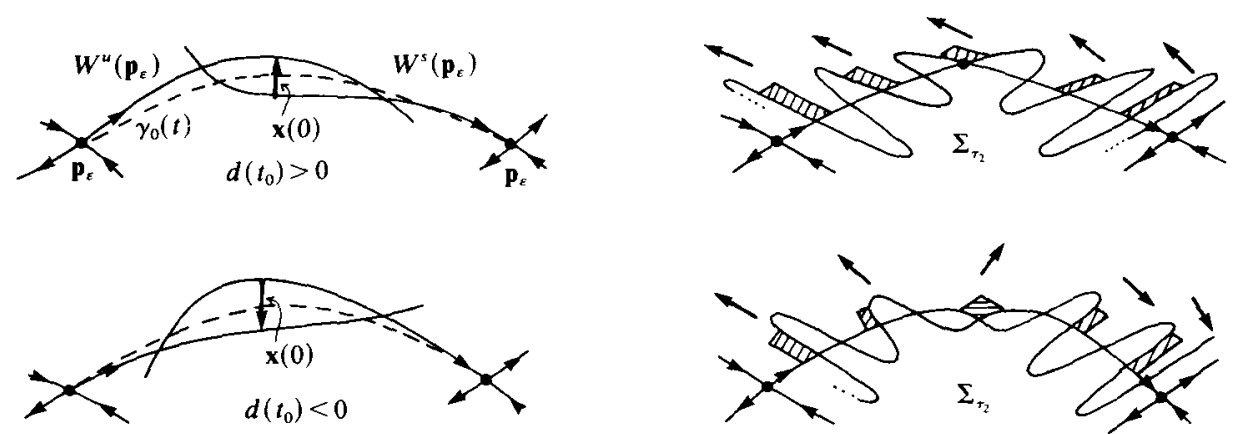

(a) $d\left(t_{0}\right)>0$ and $<0$.

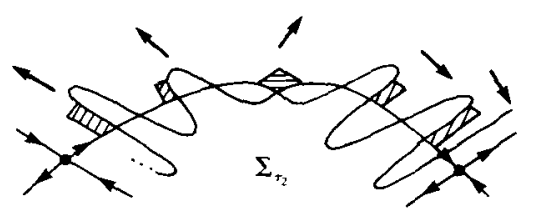

(b) order preserving and non order preserving orbits.

FIGURE 6. Homoclinic geometry.

To deal with the periodic (subharmonic) orbits, we perturb from resonant orbits with energies $h=h(m, n)$ determined uniquely for each $m, n \in \mathbb{Z}^{+}$and $(m, n)=1$ from the resonance relation

$$
\tau(h)=2 \sqrt{\frac{2}{h(m, n)}} K\left(\frac{2}{h(m, n)}\right)=\frac{2 \pi m}{\omega n} .
$$

Substituting $h(m, n)$ into the ' + ' rotary orbits (5.8) and substituting the result into the Melnikov integral (5.4), we obtain

$$
\begin{aligned}
M^{m / n}\left(t_{0} ; \bar{\nu}, \bar{\beta}, \bar{\delta}, \omega\right)= & \int_{-\pi}^{\pi}[\bar{\nu}-\bar{\delta} \sqrt{2 h-2(1-\cos \phi)}] d \phi \\
& +\sqrt{2 h} \bar{\beta} \int_{-\infty}^{\infty} \operatorname{dn}\left(\sqrt{\frac{h}{2}} t \mid \frac{2}{h}\right) \cos \omega\left(t+t_{0}\right) d t
\end{aligned}
$$

where $h=h(m, n)$ and we have used the same change of variables $r d t=d \phi$ as in (5.10). (5.16) simplifies to

$$
\begin{aligned}
M^{m / n}\left(t_{0} ; \bar{\nu}, \bar{\beta}, \bar{\delta}, \omega\right)= & \int_{-\infty}^{\infty}\left[\bar{\nu}-\sqrt{2 h} \tilde{\delta} \sqrt{1-2 \sin ^{2}\left(\frac{\phi}{2}\right)}\right] d \phi \\
& +\sqrt{2 h} \bar{\beta} \int_{-\infty}^{\infty}\left[\operatorname{dn}\left(\sqrt{\frac{h}{2}} t \mid \frac{2}{h}\right) \cos \omega t\right] d t \cos \omega t_{0}
\end{aligned}
$$


which can be evaluated to yield

$$
M^{m / n}\left(t_{0} ; \bar{\nu}, \bar{\beta}, \bar{\delta}, \omega\right)=2 \pi \bar{\nu}-8 \bar{\delta} \sqrt{\frac{h}{2}} E\left(\frac{2}{h}\right)+2 \pi \beta \operatorname{sech}\left[\frac{\pi m K^{\prime}(2 / h)}{K(2 / h)}\right] \cos \omega t_{0},
$$

for $n=1$ and

$$
2 \pi \bar{\nu}-8 \bar{\delta} \sqrt{\frac{h}{2}} E\left(\frac{2}{h}\right),
$$

for $n>1$. Here the Fourier series representation of the elliptic function

$$
\operatorname{dn}\left(\sqrt{\frac{h}{2}} t \mid \frac{2}{h}\right)=\frac{\pi}{K(2 / h)}\left\{\frac{1}{2}+\sum_{j=1}^{\infty} \operatorname{sech}\left[\frac{j \pi K^{\prime}(2 / h)}{K(2 / h)}\right] \cos \frac{\omega n j t}{m}\right\},
$$

has been used with $h=h(m, n)$. Note that, as $m \rightarrow \infty$ with $n=1,(5.18 \mathrm{a})$ converges on $M^{\infty}$ (5.12), cf. theorem 4.6.4 of [25] and [22]. To see this more easily, we remark that the argument of the sech term of (5.18a) can be re-expressed, using (5.15) with $n=1$, as $\left[\omega \sqrt{(2 / h)} K^{\prime}(2 / h)\right]$, and that $K^{\prime}(2 / h) \rightarrow \pi^{+} / 2$ as $h \rightarrow 2^{+^{-}}$. Application of theorem 5.3 then yields the 'wedges'

$$
\bar{\beta}>\left|\frac{\pi \bar{\nu}-4 \bar{\delta} \sqrt{(h / 2)} E(2 / h)}{\pi \operatorname{sech}\left[\omega \sqrt{(2 / h)} K^{\prime}(2 / h)\right]}\right|,
$$

(which, after multiplication through by $\varepsilon$, are those of theorem 5.6) in which (5.18a) has $2 m$ simple zeros in the interval $t_{0} \in[0,2 \pi m / \omega)$, and hence in which there exist precisely 2 distinct $2 \pi m / \omega$ periodic orbits.

As above, we note that, for sufficiently small $\varepsilon$, these $n=1$ periodic orbits lie close to the order preserving parent orbits of rotation number $1 / \mathrm{m}$ (period $T(h(m, 1))=2 \pi m / \omega)$ and hence that they are also order preserving.

Remark. An extension of the analysis above (cf. [23]) shows that one of each pair of $m$-periodic orbits is of saddle-type and that the other (for sufficiently small $\varepsilon$, depending on $m$ ) is a sink. For larger $m$ the sink typically bifurcates to a saddle with negative eigenvalues, throwing off a period $2 m$ sink, and this initiates a cascade of period doubling bifurcations leading ultimately to the creation of a full shift (horseshoe) for $P_{\varepsilon}^{m}$. See $[25, \S 6.6]$ and [66]. Such shifts, for $m \geq N$, lie in the set $\Lambda$ of $\S 2$.

In figure 7 we show computations of the 'resonant wedges' in $\nu, \beta$ parameter space for $\omega, \delta$ fixed. This graph makes it clear that there exist open sets (within the

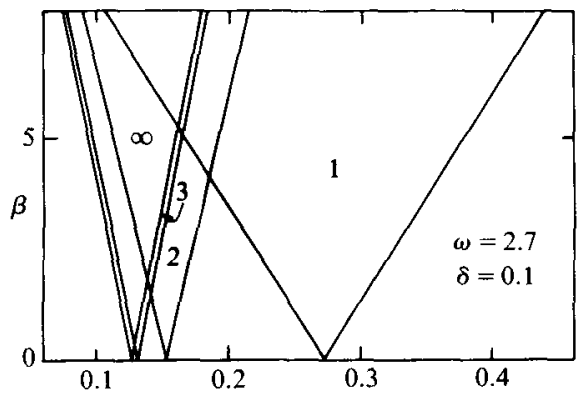

Figure 7. The 'wedges' $\beta=\beta_{m}$ for $m=1,2,3$ and the homoclinic 'wedge' $\beta=\beta_{\infty}$ for the case $\delta=0.1$, $\omega=2.7$. 
'homoclinic wedge' $\beta=|(\pi \nu-4 \delta) / \pi \operatorname{sech}(\pi \omega / 2)|)$ in which there exist order preserving $m$-periodic orbits for all $m \geq 1$ along with order preserving 1-rotary, transverse homoclinic orbits.

We remark that the boundaries $\beta=\beta_{m}$ of the parameter wedges within which $m$-periodic orbits exist accumulate on the homoclinic wedge $\beta=\beta_{\infty}$ at a geometric rate determined by the expanding eigenvalue of the saddle point $\mathbf{p}_{\varepsilon}$. This rate, $\exp (2 \pi / \omega)\left(1-(\varepsilon \delta) / 2+\sigma\left(\varepsilon^{2}\right)\right)$, is specific to the problem and is not a universal constant, cf. $[25, \S 6.6]$. In figure 8 we show typical computations of stable and

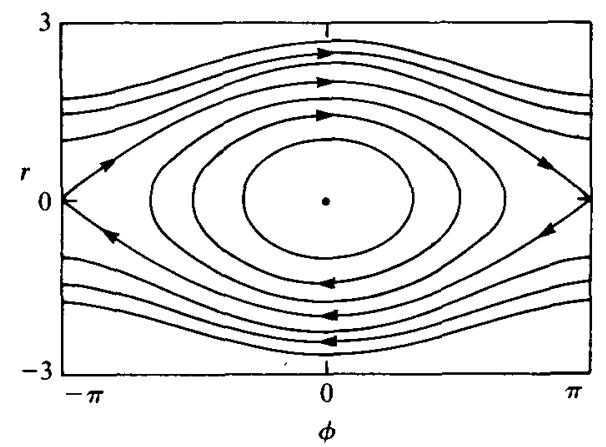

(a)

Figure 8(a). The unperturbed Poincaré map $P_{0}$.

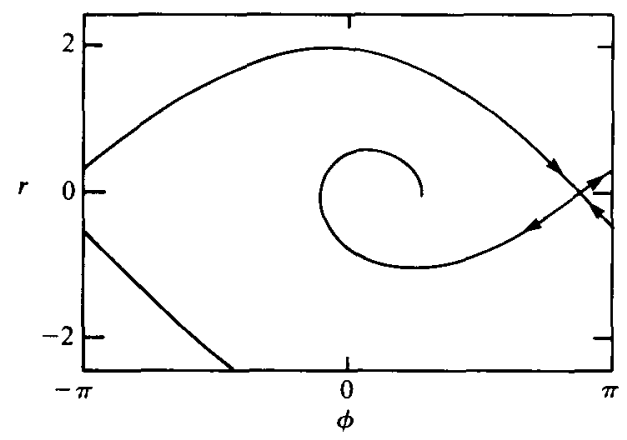

(b)

FIgURE 8(b). The stable and unstable manifolds of the fixed point $p_{\varepsilon}$ of the Poincaré map $P_{\varepsilon}$ in the case $\beta=0, \delta \approx \pi / 10, \nu=0.39025$ and $\omega=2$, for $\beta, \delta, \omega$ fixed, $\nu$ was chosen so that the upper homoclinic connection was preserved. Compare with the value $\nu=0.4$ predicted by the Melnikov function $M^{\infty}$ (eq. 5.12).

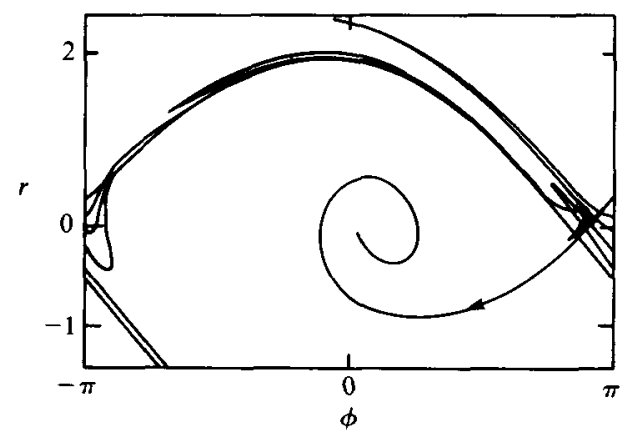

Figure $8(\mathrm{c})$. The stable and unstable manifolds of the fixed point $p_{\varepsilon}$ of the Poincare map $P_{\varepsilon}$. Here $\nu=0.396, \delta=\pi / 10, \beta=0.1$ and $\omega=2$. 
unstable manifolds for $P_{\varepsilon}$. These numerical results were obtained using a 4th order Runge-Kutta algorithm with fixed step size $\boldsymbol{h}=\mathbf{0 . 0 1}$.

5.7. Estimates on the number $N$ of iterates of $P_{\varepsilon}$ necessary to guarantee hyperbolicity for $\left.\mathbf{P}_{\varepsilon}^{N}\right|_{U}$. In proving the Smale-Birkhoff homoclinic theorem and related results, we select neighbourhoods $U$ and integers $N$ such that the map $\left.f^{N}\right|_{U}$ is uniformly hyperbolic on certain strips $\left(V_{0}, V_{1}\right.$ in $\left.\$ 2.7\right)$. The usual proofs just establish hyperbolicity for $N<\infty$ 'large enough'. In this section we show how the Melnikov function (5.3) permits us to estimate $N$ in terms of the parameters $\mu((\nu, \beta, \delta, \omega)$ here $)$. The following argument is summarized from [33, Appendix B].

Since $d\left(t_{0}\right)=\varepsilon M^{\infty}\left(t_{0} ; \mu\right) /\|f(\mathbf{x}(0))\|+\mathcal{O}\left(\varepsilon^{2}\right)$, and $\|f(\mathbf{x}(0))\|=\mathscr{O}(1)$, the angle between the tangent spaces of the manifolds $W^{u}\left(\mathbf{p}_{\varepsilon}\right), W^{s}\left(\mathbf{p}_{\varepsilon}\right)$ at a transversal intersection point $q$ corresponding to a simple zero $t_{0}=\tau$ is of $\mathscr{O}\left(\varepsilon M^{\prime}(\tau, \mu)\right)$, where ()$^{\prime}$ denotes $\partial() / \partial t_{0}$. As in $\S 2.7$, we choose a neighbourhood $U$ of $\mathbf{p}_{\varepsilon}$ and integers $k_{1}, l_{1}$ and let $V \subset f^{l_{1}}(U) \cap f^{-k_{1}}(U)$ be a neighbourhood of q. For fixed $U, k_{1}, l_{1}$, it follows that the angles between the tangent spaces of the manifolds at $f^{k_{1}}(q)$ and $f^{-l_{1}}(\mathbf{q})$ are also $\mathcal{O}\left(\varepsilon M^{\prime}\right)$ (albeit now multiplied by $k_{1}$ and $l_{1}$ dependent constants). To ensure hyperbolicity of $f^{N} \mid \Lambda_{N}$, we 'shrink $U$ and increase $k_{1}, l_{1}$ '. More precisely, we pick a smaller neighbourhood $\tilde{U} \subset U$ and integers $k_{2} \gg k_{1}, l_{2} \gg l_{1}$ such that the tangent spaces of the manifolds at $f^{k_{1}+k_{2}}(\mathbf{q})$ and $f^{-\left(l_{1}+l_{2}\right)}(\mathbf{q})$ lie at a large angle (say $>\pi / 4)$. Then the disjoint invariant conefields necessary for the establishment of a hyperbolic structure can be found (cf. $[50, \S 3],[25, \S 5.2]$ ). The integers $k_{2}$ and $l_{2}$ are estimated from the linearization $D f(x)$ of the map, which is close to the constant matrix $D f(\mathbf{p})$ for $\mathbf{x} \in U$. In fact, if the eigenvalues of $D f(\mathbf{p})$ are $0<\lambda_{2}<1<\lambda_{1}$, the estimate

$$
\left(\begin{array}{l}
t_{1} \\
t_{2}
\end{array}\right)_{i+1}=\left[\begin{array}{cc}
\lambda_{1} & 0 \\
0 & \lambda_{2}
\end{array}\right]\left(\begin{array}{l}
t_{1} \\
t_{2}
\end{array}\right)_{i}
$$

shows that a tangent vector $\left(\begin{array}{c}\varepsilon M^{\prime} C\left(k_{1}\right) \\ 1\end{array}\right)$ will be mapped to a vector in the $\left(\begin{array}{l}1 \\ 1\end{array}\right)$ direction in $k_{2}=\left[\ln \left(1 / M^{\prime} C\left(k_{1}\right)\right] /\left[\ln \left(\lambda_{1} / \lambda_{2}\right)\right]\right.$ iterates. A similar estimate holds for $l_{1}$. The final estimate of the number of iterations necessary to obtain a hyperbolic structure is therefore:

$$
N=k_{1}+k_{2}+l_{1}+l_{2}=\left(k_{1}+l_{1}\right)+\frac{2 \ln \left(1 / \varepsilon M^{\prime}(\tau, \mu) C\left(k_{1}, l_{2}\right)\right)}{\ln \left(\lambda_{1} / \lambda_{2}\right)} .
$$

For the Josephson problem, a standard computation using the unperturbed flow shows that the eigenvalues of the Poincaré map $D P_{\varepsilon}\left(\mathbf{p}_{\varepsilon}\right)$, linearized at the perturbed saddle point, are $\lambda_{1,2}=e^{ \pm(2 \pi / \omega)}+\mathcal{O}(\varepsilon)$. Using this in (5.22) we obtain

$$
N=C_{1}+\frac{\omega}{2 \pi} \ln \left(\frac{1}{C_{2} M^{\prime}(\tau ; \mu)}\right) .
$$

From (5.12), the zeros $t_{0}=\tau$ of $M^{\infty}$ are given by

$$
\cos \omega t_{0}=\frac{4 \bar{\delta}-\pi \bar{\nu}}{\pi \bar{\beta} \operatorname{sech}(\pi \omega / 2)},
$$

and using $\varepsilon M^{\prime}=-\varepsilon 2 \pi \omega \bar{\beta} \operatorname{sech}(\pi \omega / 2) \sin \omega t_{0}$ and dividing out $\varepsilon$, via $\varepsilon \bar{\beta}=\beta$, etc. 
we find

$$
\varepsilon M^{\prime}(\tau, \mu)=2 \omega \sqrt{\pi^{2} \beta^{2} \operatorname{sech}^{2}(\pi \omega / 2)-(\pi \nu-4 \delta)^{2}} .
$$

Thus the final estimate we obtain is

$$
N=C_{1}+(\omega / 2 \pi) \ln \left[1 /\left(2 \pi \omega C_{2} \operatorname{sech}(\pi \omega / 2) \sqrt{\beta^{2}-\beta_{\infty}^{2}}\right)\right],
$$

where $\beta_{\infty}=\beta_{\infty}(\nu, \delta, \omega)=|(\pi \nu-4 \delta) / \pi \operatorname{sech}(\pi \omega / 2)|$ is the critical value which the periodic force amplitude $\beta$ must exceed to guarantee transverse homoclinic points, and $C_{1}$ and $C_{2}$ are constants independent of the perturbation parameters $\nu, \beta, \delta, \omega$.

The next result returns explicitly to our characterization of the attracting set and provides a summary of what we have found so far.

5.8. Theorem. For $\beta>\beta_{\infty}=|(\pi \nu-4 \delta) / \pi \operatorname{sech}(\pi \omega / 2)|$ and $\nu, \beta, \delta$ all positive, sufficiently small, and of the same order, the attracting set $\mathscr{A}=\bigcap_{n=0}^{\infty} P_{\varepsilon}^{N}(B)$ for the Poincaré map of the Josephson equation (5.1) satisfies the following:

(i) $\mathscr{A}$ contains an invariant Cantor set $\Omega$ on which $P_{\varepsilon}$ is conjugate to a subshift of finite type.

(ii) $\Omega$ contains orbits having all rotation numbers in the closed interval $[0,1 / N]$, where $N=\mathcal{O}\left(\ln \left[1 /\left(\operatorname{sech}(\pi \omega / 2) \sqrt{\beta^{2}-\beta_{\infty}}\right)\right]\right)$.

(iii) $\Omega$ contains uncountably many disjoint invariant Cantor sets composed of orbits having rotation number $\alpha$ for each $\alpha \in[0,1 / N] \backslash \mathbb{Q}$.

(iv) $\Omega \subset A$ can be chosen so that, for each such irrational $\alpha$, at least one of these Cantor sets is order preserving ( $a$ 'dissipative' Aubry-Mather set). Moreover, at least one periodic orbit of each rotation number $p / q \in[0,1 / N]$ is likewise order preserving (a Birkhoff orbit).

(v) $\Omega$ contains uncountably many points for which rotation numbers do not exist. In any neighbourhood of a point in $\Omega$ there are points with all rotation numbers in $[0,1 / N]$ along with points whose rotation numbers do not exist.

Proof. Most of the assertions above follow directly from the results of $\$ \$ 2-4$ and the Melnikov computations of $\S 5.6$. In particular, the hypotheses imply (via theorem 5.6) that the Poincaré map $P_{\varepsilon}$ has an order preserving 1-rotary transverse homoclinic orbit. This orbit clearly lies within the trapping region $B$, and hence is contained in $\mathscr{A}$ (proposition 5.1). For conclusions (i), (ii), (iii) and (v), $\Omega$ can be taken to be the Cantor set $\Lambda$ of proposition 2.7 and theorems 2.8 and 3.1. In 2.7 we actually proved that $\left.P_{\varepsilon}^{N}\right|_{\Lambda^{N}}$ is conjugate to a full shift on two symbols, but a subshift for $\left.P_{\varepsilon}\right|_{\Lambda}$ is easily demonstrated as in $[25, \S 5.3]$. For conclusion (iv) we take $\Omega$ to be the Cantor set $\Lambda^{\prime \prime}=\Lambda^{\prime} \cup\left(\cup_{j=1}^{N-1} P_{\varepsilon}^{j}\left(\Lambda^{\prime} \cap V_{1}\right)\right)$, i.e. the Cantor set for $P_{\varepsilon}$ corresponding to that for the induced map

$$
\left.g\right|_{U}=\left\{\begin{array}{c}
\left.P_{\varepsilon}\right|_{V_{0}^{\prime}} \\
\left.P_{\varepsilon}^{N}\right|_{V_{1}}
\end{array}\right\}
$$

of $\$$ 4.2. Then, since our homoclinic orbit is order preserving, proposition 4.6 yields order preserving Cantor sets.

Remarks. Although it provides information about large collections of orbits lying within the attracting set $\mathscr{A}$, theorem 5.8 is far from a complete global description 
of $\mathscr{A}$, for the sets $U$ and $\left\{f^{j}\left(V_{1}\right)\right\}_{j=1}^{N-1}$ with which the Cantor sets $\Lambda^{N}, \Lambda, \Lambda^{\prime}$ (and hence $\Omega$ ) are constructed are carefully chosen small neighbourhoods of certain special fixed and homoclinic points. However, extensions of Melnikov theory described in [23], [24] and [34] and applied there to Duffing's equation, permit one to obtain a somewhat more complete characterization of the attracting set. It is possible to prove, for example, that open sets of $(\nu, \beta, \delta)$ values near $(0,0,0)$ exist such that the attracting set contains at least $M$ stable periodic orbits of periods $N, \ldots, N+M-1$, where $N, M$ are pre-assigned positive integers. In such cases, the attracting set is not indecomposable and so we do not have a strange attractor; cf. theorem 4.9 of [24]. One can also show that the attracting set is the closure of the unstable manifold of one of the periodic saddle points contained in it.

We end this section with examples which illustrate that the rotation set for a dissipative diffeomorphism of the annulus can be rather complicated, in contrast to the cases of maps of the circle and area preserving monotone twist maps of a (closed) annular region. In both these cases it is known that the rotation set - the collection $\left\{\rho(x) \mid x \in S^{1}\right.$ (resp. A)\} of all rotation numbers of points in the circle or the annulus respectively - is a closed interval ([52], [36], [38]). One might conjecture that the same holds true, under appropriate conditions, for a Birkhoff attracting set. (Birkhoff's [10] original example only showed that the attracting set can contain orbits with two different rotation numbers.) However, the following examples demonstrate that this conjecture is false although it is true for (indecomposable) Birkhoff attractors.

5.9. Proposition. For $\nu>4 \delta / \pi, \beta=0$ and $\nu, \delta>0$ uniformly small, the attracting set $\mathscr{A}$ of the Josephson junction has a rotation set $\{0\} \cup\{\alpha\}$, where $\alpha=\alpha(\nu, \delta) \rightarrow 0$ as $\nu \rightarrow 4 \delta / \pi$; i.e. the rotation set has empty interior.

Sketch of proof. We apply conventional phase plane and linearization techniques, together with the Melnikov perturbation method, to the autonomous system (5.1) with $\beta=0$. We conclude that, under the hypotheses above, the $\omega$-limit set consists of three disjoint components: a sink and a saddle point at $(s, 0)$ and $(p, 0)$ whose $s$ and $p$ are the two roots of $\phi=\sin ^{-1}(\nu)$, and an attracting closed curve (limit cycle) near the level set $F^{-1}(h)$ of the unperturbed Hamiltonian system, where $h=h(\nu, \delta)$ solves

$$
\sqrt{\frac{h}{2}} E\left(\frac{2}{h}\right)=\frac{\pi v}{4},
$$

(cf. equation (5.18) with $\beta=0$ ). The rotation number $\alpha(\nu, \delta)$ is close to the unperturbed rotation number

$$
\alpha(h)=\frac{2 \pi}{\omega \tau(h)}>0,
$$

where $\tau(h)=\left.2 \sqrt{(2 / h)} K(2 / h)\right|_{h=h(\nu, \delta)}$ is the period of unperturbed orbits in the level set $F^{-1}(h)$. Since all points in $A$ eventually enter $B$ and approach a component of the $\omega$-limit set, a point has (forward) rotation number either 0 (if it approaches either fixed point) or $\alpha$ (if it approaches the limit cycle). In this case the rotation number is defined for all points $x \in A$. 
5.10. Proposition. Suppose that $\nu, \beta, \delta>0$ are all small and of the same order and that

$\beta>\min \left\{\beta_{\infty}=\left|\frac{\pi \nu-4 \delta}{\pi \operatorname{sech}(\pi \omega / 2)}\right|, \quad \beta_{m}=\left|\frac{\pi \nu-4 \delta \sqrt{(h / 2)} E(2 / h)}{\pi \operatorname{sech}\left(\omega \sqrt{(2 / h)} K^{\prime}(2 / h)\right)}\right|\right\}, \quad \beta<\beta_{m-1}$, where $h=h(m)$ solves $\sqrt{2 / h} K(2 / h)=\pi m / \omega$. Then the rotation set of the Josephson attractor is contained in the interval $[0,1 / m]$ and contains the interval $[0,1 / N]$, where $N \sim \ln \left(1 / \sqrt{\beta^{2}-\beta_{\infty}^{2}}\right)$. It also contains finitely many isolated points $\{1 /(m+j)\}$, $0 \leq j \leq J$ with $J<N-m$.

Sketch of proof. Melnikov theory and the averaging theorem ([27]), as in [23], permit us to prove the existence of one sink and one saddle of each period $m+j$ in some interval $j \in[0, J]$, provided that $\min \left(\beta_{\infty}, \beta_{m}\right)<\beta<\beta_{m+1}$ and $\beta, \nu, \delta$ are all sufficiently small. We remark that, to guarantee stability types of the $m+j$ periodic points and not merely existence, one must take $\varepsilon<\varepsilon(J)$ small enough, depending explicitly on $J$. A phase plane analysis of the averaged equation (cf. [23, $\S \S 10.3 .3$, 10.6.1 and figs. 10.12-13]) then reveals that the stable and unstable manifolds of each $m+j$-periodic saddle do not mutually intersect (except at the saddle points themselves), and that the $\omega$-limit set in the neighbourhood of each resonant unperturbed level curve $F^{-1}(h(m+j))$ consists of the $m+j$-periodic saddle and sink alone. Thus there exist points in $A$ asymptotic to periodic orbits of periods 0 (the fixed points near $(0,0)$ and $(\pi, 0))$, and $m, \ldots, m+J$, but there are no orbits of periods $l \in(0, m)$. Finally, since all these periodic orbits are small perturbations of order preserving orbits lying in the level sets $F^{-1}(h(m+j))$ with rotation numbers $1 / m, \ldots, 1 / m+J$, we obtain the isolated points $\{1 / m\} \cup \cdots \cup\{1 /(m+J)\}$ of the rotation set. The interval $[0,1 / N]$ is just the interval of theorem 5.8 .

We remark that generally one must take $N \gg m+J$ and that the perturbation and averaging methods cannot determine the complete structure of the Poincaré map and the rotation set between the sets $[0,1 / N]$ and $\{1 /(m+J)\} \cup \cdots \cup\{1 / m\}$. For example, there might be several disjoint closed intervals in the rotation set.

5.11. The core of the attracting set. The above examples illustrate the importance of the notion of the core of the attractor $([10])$. Let $x \in B$ be a point in the trapping region and apply the inverse map. If $f^{-k}(\mathbf{x}) \notin B$ for some $k$ and lies below $B$ we say $\mathbf{x} \in V_{1}$, if $f^{-k}(\mathbf{x})$ lies above $B$ we say $\mathbf{x} \in V_{2}$. Letting $\mathbf{x}$ range over $B$ and taking $k$ arbitrarily large (if necessary) we thus define two disjoint open sets $V_{1}, V_{2}$ bounded by pieces of the attracting set $\mathscr{A}$ and the boundaries of $B$. The core $\mathscr{C} \subseteq \mathscr{A}$ is defined as

$$
\mathscr{C}=\mathrm{Cl}\left(V_{1}\right) \cap \mathrm{Cl}\left(V_{2}\right),
$$

and $\mathscr{C}$ is generally smaller than $\mathscr{A}$. (In proposition $5.9 \mathscr{C}$ is the closed curve alone, while $\mathscr{A}=$ (closed curve $) \cup\left(\mathrm{Cl}\left(W^{u}(\mathbf{p})\right)\right.$.) Le Calvez [40] and Casdagli [16] have recently (independently) proved that the rotation set of the core is a closed interval.

We remark that the difficulties of multiple attractors (sinks which lie in $\mathscr{A} \backslash \mathscr{C}$ ) of proposition 5.10 arise in the 'almost area preserving' limit. We expect that strongly contracting 'almost one dimensional' annulus maps will often have coincident cores and attractors. 


\section{Conclusions}

In this paper we have attempted to relate several different areas of dynamical systems theory. We have shown how transversal homoclinic orbits in dissipative diffeomorphisms of the annulus imply the existence of Smale horseshoes which can be constructed in such a way that their symbolic dynamical description preserves information on rotation numbers. A more subtle embedding permits one to construct order preserving orbits and Cantor sets which are the 'dissipative' analogue of the Aubry-Mather invariant sets of area preserving monotone twist, maps along with uncountable sets of badly ordered Cantor sets. We have applied our general theorems on annulus maps to a specific example of a Poincaré map arising in a forced non-linear oscillator: the Josephson junction equation. Using Melnikov perturbation methods, we have checked the hypotheses of our theorems and thus provided a partial characterization of the structure of the Birkhoff attracting set for the Josephson problem. In doing so, we point out that the orbit structure and consequently the structure of the rotation set for a dissipative diffeomorphism of the annulus can be considerably more complex than those for circle maps or area preserving monotone twist maps.

Acknowledgements. We would like to thank John Hubbard, David Rand and Peter Veerman for comments, criticisms and corrections during the course of this work. Thanks are also due to Philip Boyland, who drew our attention to Hedlund's paper, and to the referee for trimming part of our fat.

Research partially supported by NSF under MEA 80-17570 and MEA 84-02069.

\section{REFERENCES}

[1] A. A. Abidi \& L. O. Chua. On the dynamics of Josephson junction circuits. Electronic Circuits and Systems 3 (1979), 186-200.

[2] V. I. Arnold. Geometrical Methods in the Theory of Ordinary Differential Equations. Springer-Verlag: New York, Heidelberg, Berlin, 1982.

[3] D. G. Aronson, M. A. Chory, G. R. Hall \& R. P. McGeehee. Bifurcations from an invariant circle for two parameter families of maps of the plane: a computer assisted study. Comm. Math Phys. 83 (1982), 303-354.

[4] S. Aubry. The twist map, the extended Frankel-Kontorova model, and the Devil's staircase. Physica 7 (1983), 240-258.

[5] S. Aubry \& P. LeDaeron. The discrete Frenkel-Kontorova model and its extensions. Physica 8D (1983), 381-422.

[6] V. N. Belykh, N. F. Pedersen \& O. H. Soerensen. Shunted Josephson Junction model, I-The autonomous case and II-the non-autonomous case. Phys. Rev. B. 16 (1977), 4853-4871.

[7] G. D. Birkhoff. Proof of Poincaré's geometric theorem. Trans. Amer. Math. Soc. 14 (1913), $14-22$.

[8] G. D. Birkhoff. Dynamical Systems. Amer. Math. Soc. Colloq. Publ. 9, Providence, R.I., 1927 (reissued 1966, 1981).

[9] G. D. Birkhoff. Sur l'existence de regions d'instabilité en Dynamique. Annales de l'Inst. Henri Poincaré 2 (1932), 369-386.

[10] G. D. Birkhoff. Sur quelques courbes fermées remarquables. Bull. de la Soc. Mathem. de France 60 (1932), 1-26.

[11] T. Bohr, P. Bak and M. H. Jensen. Transition to chaos by interaction of resonances in dissipative systems: II. Josephson junctions, charge density waves and standard maps. Preprint, Cornell University, 1984.

[12] P. Boyland. Bifurcations of circle maps, Arnold tongues, bistability and rotation intervals. Preprint, Boston University, 1983. 
[13] P. L. Boyland \& G. R. Hall. Invariant circles and the order structure of periodic orbits in monotone twist maps. Preprint, Boston University.

[14] P. F. Byrd \& M. D. Friedman. Handbook of Elliptic Integrals for Scientists and Engineers. SpringerVerlag: New York, Heidelberg, Berlin, 1971.

[15] M. L. Cartwright \& F. E. Littlewood. On nonlinear differential equations of the second order I: The equation $\ddot{y}+k\left(1-y^{2}\right) \dot{y}+y=b \lambda k \cos (\lambda t+a), k$ large. J. Lond. Math. Soc. 20 (1945), 180-189.

[16] M. Casdagli. Periodic orbits for Birkhoff attractors. Preprint, Univ. of Warwick, 1985.

[17] A. Chenciner. Bifurcations de difféomorphismes de $\mathbb{R}^{2}$ au voisiange d'un point fixe elliptique. Les Houches Summer School Proceedings, ed. R. Helleman, G. Iooss, North Holland, 1983.

[18] A. Chenciner. Bifurcations de points fixes elliptiques, I-Courbes invariantes, et II Orbites périodiques et ensembles de Cantor. To appear in Publ. Math. IHES.

[19] A. Chenciner, J. M. Gambaudo \& C. Tresser. Une remarque sur la structure des endomorphismes de degre 1 du cercle. C.R.A.S. Paris, 299, Serie I, 5 (1984), 145-148.

[20] A. Chenciner, J. M. Gambaudo \& C. Tresser. Une remarque sur les familles d'endomorphismes de degré un du cercle. C.R.A.S., Paris, 299, Serie I, 15, (1984), 771-773.

[21] B. V. Chirikov. A universal instability of many dimensional oscillator systems. Physics Reports 52, (1979), 263-379.

[22] S. N. Chow, J. K. Hale \& J. Mallet-Paret. An example of bifurcation to homoclinic orbits. J. Diff. Eqns. 37 (1980), 351-373.

[23] B. D. Greenspan \& P. J. Holmes. Homoclinic orbits, subharmonics, and global bifurcations in forced oscillations. Chapter 10,pp. 172-214 in Nonlinear Dynamics and Turbulence, ed. G. Barenblatt, G. Iooss and D. D. Joseph. Pitman: London, 1983.

[24] B. D. Greenspan \& P. J. Holmes. Repeated resonance and homoclinic bifurcation in a periodically forced family of oscillators. SIAM J. on Math. Analysis 15 (1984), 69-97.

[25] J. Guckenheimer \& P. J. Holmes. Nonlinear Oscillations, Dynamical Systems and Bifurcations of Vector Fields. Springer Verlag: New York, 1983.

[26] J. Guckenheimer \& R. F. Williams. Structural stability of Lorenz attractors. Publ. Math. IHES 50 (1979), 59-72.

[27] J. K. Hale. Ordinary Differential Equations. Wiley: New York, 1969.

[28] G. R. Hall. A topological version of a theorem of Mather on twist maps. Ergod. Th. \& Dynam. Sys. 4 (1984), 585-603.

[29] G. A. Hedlund. Sturmian minimal sets. Amer. J. Math. 66 (1944), 605-620.

[30] M. R. Herman. Sur les Courbes Invariantes par les Diffeomorphismes de l'Anneau, I. Astérisque 103 104 (1983).

[31] P. J. Holmes. Space- and time-periodic perturbations of the sine-Gordon equations. In Dynamical Systems and Turbulence, D. A. Rand and L.-S. Young (eds) pp. 164-191, Springer Lecture Notes in Mathematics 898. Springer-Verlag: New York, Berlin, Heidelberg, 1981.

[32] P. J. Holmes. The dynamics of repeated impacts with a sinusoidally vibrating table. J. Sound Vibration 84 (1982), 173-189.

[33] P. J. Holmes \& J. E. Marsden. Horseshoes in perturbations of Hamiltonian systems with two degrees of freedom. Comm. Math. Phys. 82 (1982), 523-544.

[34] P. J. Holmes \& D. C. Whitley. On the attracting set for Duffing's equation I: Analytical methods for small force and damping. In Partial Differential Equations and Dynamical Systems, W. Fitzgibbon III (ed.) pp. 211-240, (1984).

[35] B. A. Huberman, J. P. Crutchfield \& N. H. Packard. Noise phenomena in Josephson junctions. Appl. Phys. Lett. 37 (1980), 750-752.

[36] R. Ito. Rotation sets are closed. Math. Proc. Camb. Phil. Soc. 89 (1981), 107-111.

[37] M. H. Jensen, P. Bak \& T. Bohr. Transition to chaos by interaction of resonances in dissipative systems: 1. Circle Maps. Preprint, Cornell University, 1984.

[38] A. Katok. Some remarks on Birkhoff and Mather twist map theorems. Ergod. Th. \& Dynam. Sys. 2 (1982), 185-194.

[39] A. Katok. Periodic and Quasi-periodic orbits for twist maps. In Proceedings, Sitges 1982, L. Garrido (ed.). Springer-Verlag: Berlin, 1983.

[40] P. LeCalvez. Existence d'orbites quasi-périodiques dans les attracteurs de Birkhoff. Preprint, Orsay, 1985.

[41] M. Levi. Qualitative analysis of the periodically forced relaxation oscillations. Mem. Amer. Math. Soc. 244, (1981). 
[42] M. Levi. Beating modes in the Josephson Junction. Preprint, Boston University, 1984.

[43] M. Levi, F. Hoppensteadt \& W. Miranker. Dynamics of the Josephson junction. Quart. Appl. Math. 35 (1978), 167-198.

[44] N. Levinson. A second order differential equation with singular solutions. Annals of Math. 50 (1949), 127-153.

[45] J. N. Mather. Existence of quasi-periodic orbits for twist homeomorphisms of the annulus. Topology 21 (1982), 457-467.

[46] J. N. Mather. Non-uniqueness of solutions of Percival's Euler-Lagrange Equation. Comm. Math. Phys. 86 (1982), 465-476.

[47] J. N. Mather. More Denjoy minimal sets for area preserving diffeomorphisms. Preprint, Princeton University, 1984.

[48] J. Matisoo. Josephson-type superconductive tunnel junctions and applications. IEEE Transactions on Magnetics 5 (1969), 848-873.

[49] V. K. Melnikov. On the stability of the center for time periodic perturbations. Trans. Moscow Math. Soc. 12 (1963), 1-57.

[50] J. Moser. Stable and Random Motions in Dynamical Systems. Princeton University Press: Princeton, NJ, 1973.

[51] S. E. Newhouse. Lectures on dynamical systems. In 'Dynamical Systems' ed. J. K. Moser. Birkhauser: Boston, 1980.

[52] S. Newhouse, J. Palis \& F. Takens. Bifurcations and stability of families of diffeomorphisms. Publ. Math. I.H.E.S. 57 (1983), 5-72.

[53] M. Odyniec \& L. O. Chua. Josephson junction circuit analysis via integral manifolds. IEEE Transactions on Circuits and Systems CAS-30 (1983).

[54] J. Palis. On Morse Smale dynamical systems. Topology 8 (1969), 385-405.

[55] 1. C. Percival. Variational principles for invariant tori and cantori. In Symp. on Nonlinear Dynamics and Beam-Beam Interactions, M. Month and J. C. Herrara (eds.). Amer. Inst. Phys. Conf. Proc. 57, 310-320, 1980.

[56] H. Poincaré. Mémoire sur les courbes définies par les équations différentielles I-IV, (Oeuvre I). Gauthier Villars: Paris, 1880-1890.

[57] H. Poincaré. Sur les équations de la dynamique et le problème de trois corps. Acta. Math. 13 (1890), 1-270.

[58] H. Poincaré. Les Methodes Nouvelles de la Mécanique Celeste (3 Vols.) Gauthier-Villars: Paris, 1899.

[59] F. M. A. Salam \& S. S. Sastry. Dynamics of the forced Josephson junction circuit: the regions of chaos. To appear in IEEE Transactions on Circuits and Systems.

[60] J. A. Sanders \& R. Cushman. Limit cycles in the Josephson Equation. Rapport No. 264, (1984). Subfaculteit Wiskunde et Informatica, Vrije Universiteit, Amsterdam.

[61] S. Smale. Diffeomorphisms with many periodic points. In Differential and Combinatorial Topology, ed. S. S. Cairns, pp. 63-80. Princeton University Press: Princeton, N.J., 1963.

[62] S. Smale. Differentiable dynamical systems. Bull. Amer. Math. Soc. 73 (1967), 747-817.

[63] S. Smale. The Mathematics of Time: Essays on Dynamical Systems, Economic Processes and Related Topics. Springer-Verlag: New York, Heidelberg, Berlin, 1980.

[64] C. T. Sparrow. The Lorenz Equations: Bifurcations, Chaos and Strange Attractors. Springer-Verlag: New York, Heidelberg, Berlin, 1982.

[65] P. Veerman. Symbolic dynamics and rotation numbers. To appear in Physica A (1986).

[66] J. A. York \& K. T. Alligood. Cascades of period-doubling bifurcations: a prerequisite for horseshoes. Bull. Amer. Math. Soc. 9 (1983), 319-322.

[67] E. Zehnder. Homoclinic points near elliptic fixed points. Comm. Pure. Appl. Math. 26 (1973), $141-182$. 